Check for updates

Cite this: RSC Adv., 2017, 7, 39502

\title{
Highly efficient, one-pot synthesis of novel bis- spirooxindoles with skeletal diversity via sequential multi-component reaction in PEG-400 as a biodegradable solvent $\uparrow$
}

\author{
Ensieh Safari, Ammar Maryamabadi and Alireza Hasaninejad (D)* \\ A green and efficient one-pot, sequential, multi-component protocol has been developed for the synthesis \\ of some novel symmetrical bis-spirooxindole derivatives from the reaction of isatins, dihalides, malono \\ derivatives and $\mathrm{C}-\mathrm{H}$ activated carbonyl compounds or ketene aminal derivatives in the presence of \\ potassium carbonate $\left(\mathrm{K}_{2} \mathrm{CO}_{3}\right)$ in polyethylene glycol 400 (PEG-400) as a biodegradable polymeric \\ solvent at room temperature. In this research, a variety of bis-spiro-indoline-pyranopyranes, bis-spiro- \\ indoline-chromenes, bis-spiro-indoline-pyrido-pyrimidines, bis-spiro-indoline-imidazo-pyridines and \\ bis-spiro-indoline-pyridines were obtained with excellent yields within short reaction time and without \\ chromatographic separation.
}

Received 29th May 2017

Accepted 6th August 2017

DOI: $10.1039 / \mathrm{c} 7 \mathrm{ra06017c}$

rsc.li/rsc-advances

widely in bio-conjugates as tools for diagnostics and in drug

\section{Introduction}

Public concern over the effects of chemical release into the environment has grown steadily due to the potential threats to human health and indirect harm arising through disruptions of natural ecosystems. ${ }^{1}$ The introduction of green processes into chemistry has helped to reduce chemical related impact on human health and the environment. ${ }^{2}$ Since most organic reactions occur in the liquid phase, selection of solvent is one of the most active areas of research in green chemistry, because it makes up by far the greatest proportion of waste and a significant part of the hazard issues and energy intensity of a process. ${ }^{3,4}$ Green solvents should meet some basic requirements such as low toxicity, non-flammability, nonmutagenicity, non-volatility and widespread availability. ${ }^{5,6}$ Recently, polyethylene glycol (PEG) has been found to be a novel, powerful and interesting green solvent system. ${ }^{7,8}$ PEG covers many requirements of a green solvent such as thermal stability over a wide range of temperatures, recovery, nonexplosiveness, commercial availability and non-toxicity. ${ }^{8,9}$ PEGs are readily soluble in polar solvents such as water, DMF and methanol but are insoluble in non-polar solvents such as diethyl ether and $n$-hexane; this unequalled property permits the recovery of PEGs by precipitation and filtration, which is extremely significant in soluble polymer-supported chemistry. Furthermore, PEG is a biological polymer that has been used

Department of Chemistry, Faculty of Sciences, Persian Gulf University, Bushehr, 7516913817, Iran.E-mail: a_hasaninejad@yahoo.com

$\dagger$ Electronic supplementary information (ESI) available. See DOI: $10.1039 / \mathrm{c} 7 \mathrm{ra06017 \textrm {c }}$ delivery. ${ }^{10,11}$

Development of efficient chemical reaction sequences in green media that provide a molecular diversity and structural complexity with a minimum number of synthetic steps could be major challenge. ${ }^{12}$ As a solution for these challenges, multicomponent reactions (MCRs) are considered to be one of the most efficient strategies in modern organic synthesis and employing green solvents may influence the course of reaction, yields, selectivity and the reaction rates. ${ }^{13}$ MCRs are well known for their simplicity of operation, reduction of isolation and purification steps, and minimization of energy, time, costs, and waste production. ${ }^{14-17}$ The efforts to combine the advantages of MCRs and eco-friendly solvents through conducting MCRs in such solvents has been comprehensively reviewed. ${ }^{18}$ Conducting MCRs in water, ${ }^{19}$ ionic liquids, ${ }^{20}$ polyethylene glycol (PEG), ${ }^{21}$ super critical carbon dioxide $\left(\mathrm{scCO}_{2}\right),{ }^{22}$ bio-derived solvents, ${ }^{23}$ perfluorinated solvents ${ }^{24}$ and neat system $\mathrm{s}^{25}$ has attracted more attention in recent decade.

Isatin and bis-isatin spiro derivatives (including bisspirooxindoles) have been synthesized using different methodologies and have been utilized successfully in many biological processes as antimicrobial, anticancer and enzyme inhibitor agents. ${ }^{26-28}$ However, many of the synthetic routes toward bis-spirooxindoles include the use of toxic solvents and catalysts or they have been conducted in harsh conditions like refluxing for long times. ${ }^{29-31}$ Furthermore, biological studies demonstrate that the presence of an aliphatic chain in a molecule decreases the polarity and lead to increases in lipophilicity of the compound. Therefore, this property enables the compound to cross the biological membrane which cause 


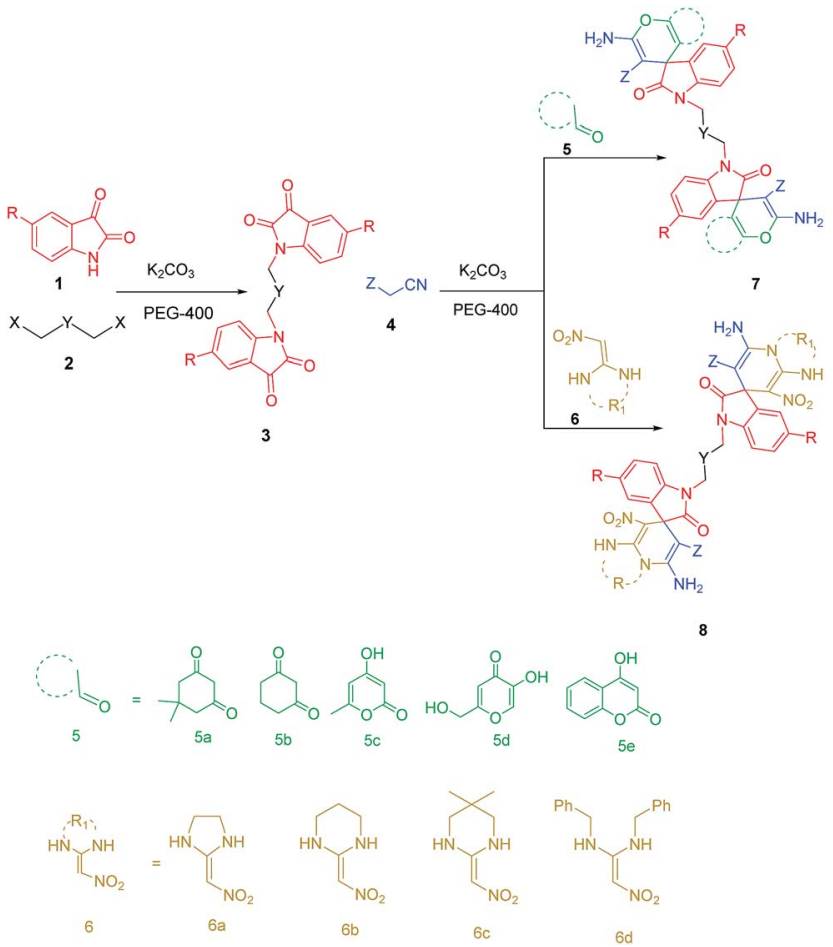

Scheme 1 The one-pot, multi-component synthesis of bis-spirooxindoles in PEG-400/ $\mathrm{K}_{2} \mathrm{CO}_{3}$ at room temperature.

increasing the biological activity of the compound. On the basis of the above hypothesis and according to the biological activity of spirooxindoles, ${ }^{32}$ we would like to report an efficient one-pot, multi-component synthesis of novel bis-spirooxindole derivatives in green medium (Scheme 1).

\section{Results and discussion}

Initially, the sequential condensation of isatin $1 \mathrm{a}(2 \mathrm{mmol}), 1,6-$ dibromohexane $2 \mathbf{a}(1 \mathrm{mmol})$, malononitrile $4 \mathbf{a}(2 \mathrm{mmol})$ and dimedone $5 \mathbf{a}$ ( $2 \mathrm{mmol}$ ) (Scheme 2) was investigated to find an

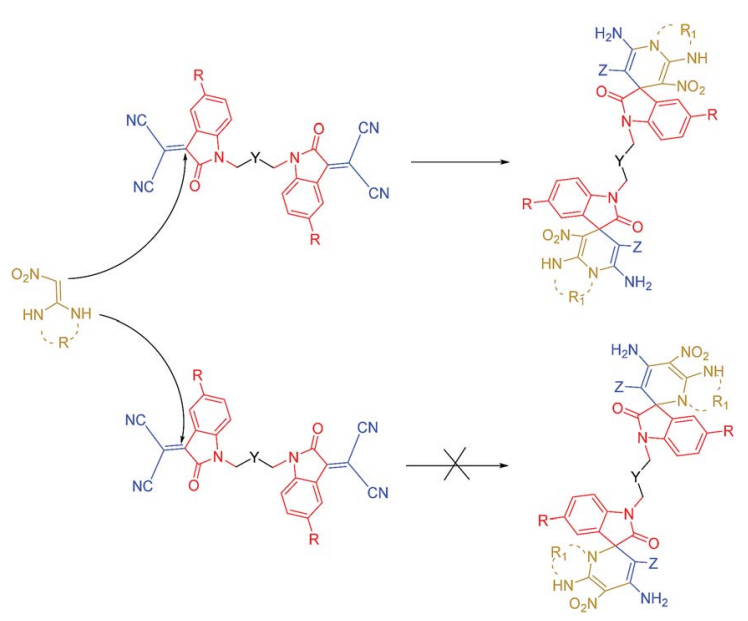

Scheme 2 Regioselectivity in ketene aminal reactions with bis-isatinmalononitrile aggregate. appropriate reaction medium for the synthesis of bisspirooxindoles. Different solvents, catalysts and reaction temperatures were explored in this investigation. Water, methanol, ethanol, acetonitrile, DMF, DMSO and PEG-400 were examined as solvent and the effects of temperature, the type and the amount of catalyst have been checked (Table 1). After this vast study, the results have depicted that the highest value for yield of desired bis-spirooxindole has been obtained in PEG-400 in the presence of $2 \mathrm{mmol}$ of $\mathrm{K}_{2} \mathrm{CO}_{3}$ at room temperature. Initially, this reaction was carried out in PEG-400 without any catalyst at room temperature. It was observed that the reaction did not proceed even after 24 hours (Table 1, entry 1). Different catalysts including L-proline, DABCO, DBU, $\mathrm{NaHCO}_{3}, \mathrm{Na}_{2} \mathrm{CO}_{3}$,

Table 1 Synthesis of bis-spiroindoline chromene 7a through one-pot sequential condensation reaction between isatin $1 \mathrm{a}(2 \mathrm{mmol}), 1,6-$ dibromohexane $2 \mathrm{a}(1 \mathrm{mmol})$, malononitrile $4 \mathrm{a}(2 \mathrm{mmol})$ and dimedone 5 a $(2 \mathrm{mmol})$ using different amounts of basic catalysts in various solvents and temperatures

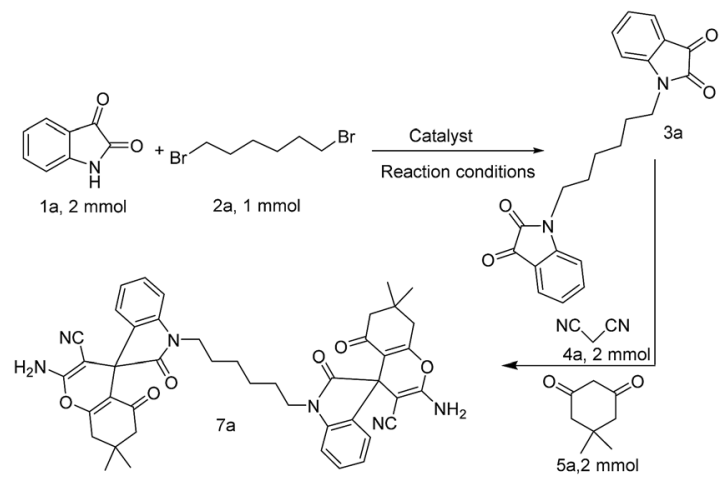

\begin{tabular}{|c|c|c|c|c|c|}
\hline Entry & $\begin{array}{l}\text { Catalyst } \\
(\mathrm{mmol})\end{array}$ & Solvent & $\begin{array}{l}\text { Temperature } \\
\left({ }^{\circ} \mathrm{C}\right)\end{array}$ & $\begin{array}{l}\text { Time } \\
\text { (h) }\end{array}$ & $\begin{array}{l}\text { Yield }^{a} \\
(\%)\end{array}$ \\
\hline 1 & - & PEG-400 & r.t. & 24 & - \\
\hline 2 & L-Proline (2) & PEG-400 & r.t. & 24 & - \\
\hline 3 & DABCO (2) & PEG-400 & r.t. & 24 & - \\
\hline 4 & DBU (2) & PEG-400 & r.t. & 24 & - \\
\hline 5 & $\mathrm{NaHCO}_{3}(2)$ & PEG-400 & r.t. & 24 & Trace \\
\hline 6 & $\mathrm{Na}_{2} \mathrm{CO}_{3}(2)$ & PEG-400 & r.t. & 24 & 42 \\
\hline 7 & $\mathrm{NaOH}(2)$ & PEG-400 & r.t. & 24 & - \\
\hline 8 & $\mathrm{CaCO}_{3}(2)$ & PEG-400 & r.t. & 24 & 30 \\
\hline 9 & $\mathrm{Cs}_{2} \mathrm{CO}_{3}(2)$ & PEG-400 & r.t. & 24 & 73 \\
\hline 10 & $\mathrm{KOH}(2)$ & PEG-400 & r.t. & 24 & - \\
\hline 11 & $\mathrm{~K}_{2} \mathrm{CO}_{3}(2)$ & PEG-400 & r.t. & 9 & 91 \\
\hline 12 & $\mathrm{~K}_{2} \mathrm{CO}_{3}(1.5)$ & PEG-400 & r.t. & 12 & 80 \\
\hline 13 & $\mathrm{~K}_{2} \mathrm{CO}_{3}(3)$ & PEG-400 & r.t. & 9 & 91 \\
\hline 14 & $\mathrm{~K}_{2} \mathrm{CO}_{3}(2)$ & PEG-400 & 50 & 9 & 90 \\
\hline 15 & $\mathrm{~K}_{2} \mathrm{CO}_{3}(2)$ & PEG-400 & 80 & 9 & 91 \\
\hline 16 & $\mathrm{~K}_{2} \mathrm{CO}_{3}$ & $\mathrm{H}_{2} \mathrm{O}$ & r.t. & 24 & - \\
\hline 17 & $\mathrm{~K}_{2} \mathrm{CO}_{3}(2)$ & $\mathrm{MeOH}$ & r.t. & 24 & - \\
\hline 18 & $\mathrm{~K}_{2} \mathrm{CO}_{3}(2)$ & EtOH & r.t. & 24 & - \\
\hline 19 & $\mathrm{~K}_{2} \mathrm{CO}_{3}(2)$ & $\mathrm{CH}_{3} \mathrm{CN}$ & r.t. & 24 & Trace \\
\hline 20 & $\mathrm{~K}_{2} \mathrm{CO}_{3}(2)$ & DMF & r.t. & 18 & 80 \\
\hline 21 & $\mathrm{~K}_{2} \mathrm{CO}_{3}(2)$ & DMSO & r.t. & 15 & 85 \\
\hline 22 & $\mathrm{~K}_{2} \mathrm{CO}_{3}$ & - & r.t. & 24 & - \\
\hline 23 & $\mathrm{~K}_{2} \mathrm{CO}_{3}(2)$ & - & 100 & 24 & - \\
\hline
\end{tabular}


$\mathrm{NaOH}, \mathrm{CaCO}_{3}, \mathrm{Cs}_{2} \mathrm{CO}_{3}, \mathrm{KOH}$ and $\mathrm{K}_{2} \mathrm{CO}_{3}$ in PEG-400 at room temperature were tested (Table 1, entries 2-11) and $\mathrm{K}_{2} \mathrm{CO}_{3}$ was the best catalyst for this transformation (Table 1, entry 11). Increasing the amount of $\mathrm{K}_{2} \mathrm{CO}_{3}$ did not improve the reaction results whereas; decreasing the amount of catalyst decreased the reaction yield (compare entries 11-13 Table 1). Increasing of the reaction temperature did not affect the reaction process (for comparison see Table 1, entries 11, 14 and 15). The model reaction was also examined in the presence of $\mathrm{K}_{2} \mathrm{CO}_{3}$ in other solvents including $\mathrm{H}_{2} \mathrm{O}, \mathrm{MeOH}$, EtOH, $\mathrm{CH}_{3} \mathrm{CN}$, DMF and DMSO. In protic solvents $\left(\mathrm{H}_{2} \mathrm{O}, \mathrm{EtOH}\right.$, and $\left.\mathrm{MeOH}\right)$ the reaction did not proceed at all, whereas change of solvent from protic to aprotic $\left(\mathrm{CH}_{3} \mathrm{CN}, \mathrm{DMF}, \mathrm{DMSO}\right)$ resulted in the formation of the desired product. This observations could be related to the solubility of starting materials and activation of catalyst in aprotic solvents. The reaction was also tested in neat conditions in the presence of $\mathrm{K}_{2} \mathrm{CO}_{3}$ at different temperatures but did not proceed even after $24 \mathrm{~h}$ (Table 1 , entries 22, 23). The reason behind this performance of PEG-400 could be related to its unique property in solving and gathering reactants and the catalyst together to react more efficiently in comparison to other solvents. ${ }^{33}$
On the basis of biological studies, the existence of two or more different heterocyclic moieties in a single molecule often enhances the biological activity dramatically. ${ }^{34,35} \mathrm{~A}$ few synthetic methods are available for the synthesis of bis-spirooxindoles derivatives. However, survey of the literature data show that the majority of these methods suffer from one or more drawbacks, such as multistep sequences, toxic solvents like DMSO and DMF, application of hazardous and toxic agents like $\mathrm{NaH}$ and long reaction times. ${ }^{29-31,36}$ Considering the aforementioned drawbacks, producing of $\mathrm{N}$-alkyl-linked bis-isatins and then obtaining the corresponding bis-spirooxindole compounds in our introduced reaction model have merits such as green solvent, non-toxic reagent, room temperature conditions, low cost, and easy work-up.

Having found the basic conditions for model reaction (PEG$400 / \mathrm{K}_{2} \mathrm{CO}_{3}$ ), we then directed our attention toward the reaction scope for the synthesis of bis-spirooxindoles and the results have been shown in Table 2 .

As shown in Table 2, all reactions proceeded efficiently, affording the corresponding products in good to excellent yields. Considering different substitutions on starting isatins, malono-derivatives and enolizable ketones, the obtained

Table 2 One-pot, multi-component synthesis of bis-spirooxindoles $(7 \mathrm{a}-7 \mathrm{u})$ in the presence of $\mathrm{K}_{2} \mathrm{CO}_{3}$ in PEG-400 at room temperature

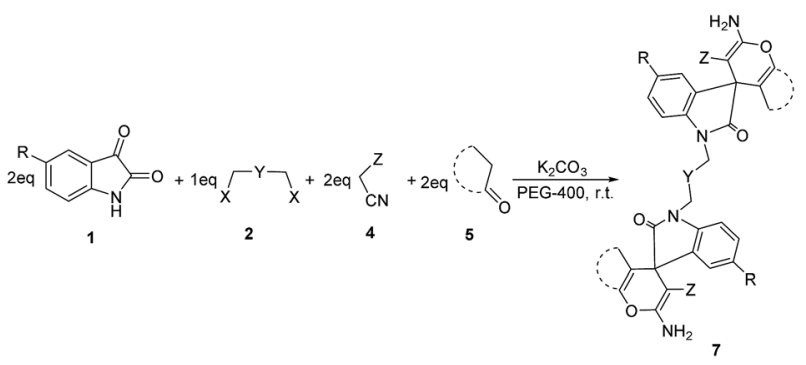

\begin{tabular}{|c|c|c|c|c|c|c|c|c|}
\hline 1 & $\mathrm{H}$ & $\left(\mathrm{CH}_{2}\right)_{4}$ & $\mathrm{Br}$ & $\mathrm{CN}$ & $5 \mathbf{a}$ & $7 \mathbf{a}$ & 9 & 91 \\
\hline 2 & $\mathrm{H}$ & $\left(\mathrm{CH}_{2}\right)_{4}$ & $\mathrm{Br}$ & $\mathrm{CO}_{2} \mathrm{Me}$ & $5 a$ & $7 b$ & 10 & 90 \\
\hline 4 & $\mathrm{Me}$ & $\left(\mathrm{CH}_{2}\right)_{4}$ & $\mathrm{Br}$ & $\mathrm{CO}_{2} \mathrm{Me}$ & $5 d$ & $7 d$ & 12 & 89 \\
\hline 5 & $\mathrm{Me}$ & $\left(\mathrm{CH}_{2}\right)_{4}$ & $\mathrm{Br}$ & $\mathrm{CO}_{2} \mathrm{Et}$ & $5 c$ & $7 e$ & 12 & 90 \\
\hline 6 & $\mathrm{Br}$ & $\left(\mathrm{CH}_{2}\right)_{4}$ & $\mathrm{Br}$ & $\mathrm{CO}_{2} \mathrm{Et}$ & $5 b$ & $7 f$ & 11 & 90 \\
\hline 9 & $\mathrm{~F}$ & $\left(\mathrm{CH}_{2}\right)_{4}$ & $\mathrm{Br}$ & $\mathrm{CO}_{2} \mathrm{Me}$ & $5 c$ & $7 \mathbf{i}$ & 9 & 92 \\
\hline 10 & $\mathrm{H}$ & $\left(\mathrm{CH}_{2}\right)_{2}$ & $\mathrm{Br}$ & $\mathrm{CO}_{2} \mathrm{Me}$ & $5 e$ & $7 \mathbf{j}$ & 10 & 90 \\
\hline 11 & $\mathrm{Me}$ & $\left(\mathrm{CH}_{2}\right)_{4}$ & $\mathrm{Br}$ & $\mathrm{CN}$ & $5 a$ & $7 \mathbf{k}$ & 9 & 89 \\
\hline 12 & $\mathrm{H}$ & $\left(\mathrm{CH}_{2}\right)_{4}$ & $\mathrm{Br}$ & $\mathrm{CO}_{2} \mathrm{Me}$ & $5 b$ & 71 & 10 & 90 \\
\hline 13 & $\mathrm{H}$ & $\left(\mathrm{CH}_{2}\right)_{4}$ & $\mathrm{Br}$ & $\mathrm{CO}_{2} \mathrm{Et}$ & $5 d$ & $7 m$ & 8 & 89 \\
\hline 14 & $\mathrm{H}$ & $\left(\mathrm{CH}_{2}\right)_{2}$ & $\mathrm{Br}$ & $\mathrm{CO}_{2} \mathrm{Et}$ & $5 c$ & $7 n$ & 9 & 88 \\
\hline 19 & $\mathrm{Me}$ & $\left(p-\mathrm{C}_{6} \mathrm{H}_{4}\right)$ & $\mathrm{Cl}$ & $\mathrm{CO}_{2} \mathrm{Et}$ & $5 a$ & $7 s$ & 10 & 88 \\
\hline 20 & $\mathrm{H}$ & $\left(p-\mathrm{C}_{6} \mathrm{H}_{4}\right)$ & $\mathrm{Cl}$ & $\mathrm{CO}_{2} \mathrm{Et}$ & $5 a$ & $7 t$ & 10 & 90 \\
\hline 21 & $\mathrm{H}$ & $\left(p-\mathrm{C}_{6} \mathrm{H}_{4}\right)$ & $\mathrm{Cl}$ & $\mathrm{CN}$ & $5 a$ & $7 \mathbf{u}$ & 9 & 91 \\
\hline
\end{tabular}

${ }^{a}$ Isolated yield. 
compounds are representative for all possible 180 possible combinations.

It is well known that the presence of indole substructure in heterocyclic compounds such as spirooxindloes, makes them biologically active. ${ }^{37-39}$ Spirooxindoles are fascinating cores that can be found in many natural active compounds with antiproliferative, antibacterial, antifungal and antiviral activities. ${ }^{40}$ Therefore, as a future plan, it is worthy to evaluate the effect of the additional spiro core on the biological activity of the obtained bis-spiro compounds by comparing with mono-spiro activities.

Table 3 Synthesis of ketene aminal derivatives

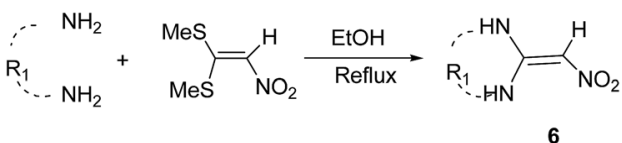

\begin{tabular}{lllll}
\hline Entry & $\mathrm{R}_{1}$ & Product & Time $(\mathrm{h})$ & Yield $^{a}(\%)$ \\
\hline 1 & $-\left(\mathrm{CH}_{2}\right)_{2}-$ & $\mathbf{6 a}$ & 2.5 & 82 \\
2 & $-\left(\mathrm{CH}_{2}\right)_{3}-$ & $6 \mathbf{b}$ & 4 & 93 \\
3 & $-\mathrm{CH}_{2} \mathrm{C}\left(\mathrm{CH}_{3}\right)_{2} \mathrm{CH}_{2}-$ & $\mathbf{6 c}$ & 2.5 & 94 \\
4 & $\mathrm{PhCH}_{2}-$ & 6d & 2 & 95
\end{tabular}

${ }^{a}$ Isolated yield.
Ketene aminals (KAs) are powerful and versatile intermediates in heterocyclic synthesis. Reactions of ketene aminals with a number of bis-electrophilic reagents have been successfully used to give five- and six-membered and fused heterocyclic compounds during the past years. ${ }^{41}$ Ketene aminals were synthesized according to literature. ${ }^{21,42}$ Briefly, 1,1bis(methylthio)-2-nitroethylene $(1 \mathrm{mmol})$ and diamines ( $1 \mathrm{mmol})$ or primary amines $(2 \mathrm{mmol})$ have been reacted in $\mathrm{EtOH}$ at reflux for 2-4 h. The precipitate has been filtered and washed by cold ethanol (82-95\% yield) (Table 3 ).

Subsequently, we expanded the scope of the performed condensation reaction by investigating the use of ketene aminals as alternatives for enolizable ketones. Under the same conditions for model reaction, the condensation reaction of isatins, dihalides, malono derivatives and ketene aminals in the presence of $\mathrm{K}_{2} \mathrm{CO}_{3}$ in PEG-400 at room temperature have been conducted, affording the corresponding products $\mathbf{8 a - 8 n}$ in good yields and in short reaction times (Table 4).

As shown in Table 4, different types of alkyl dihalides, which some of them have aromatic ring in their structure, have been effectively utilized as linker between two isatin molecules. Also isatins with electron withdrawing and electron donating substitutions on their aromatic ring have been successfully used for synthesis of these complex structures. It turned out that the electronic effects of substitution on aromatic ring of isatin has negligibly affected the time and yield of bisspirooxindole. Various aliphatic cyclic and acyclic ketene

Table 4 One-pot, multi-component synthesis of bis-spirooxindole derivatives $(8 \mathrm{a}-8 \mathrm{n})$ in the presence of $\mathrm{K}_{2} \mathrm{CO}_{3}$ in $\mathrm{PEG}-400$ at room temperature

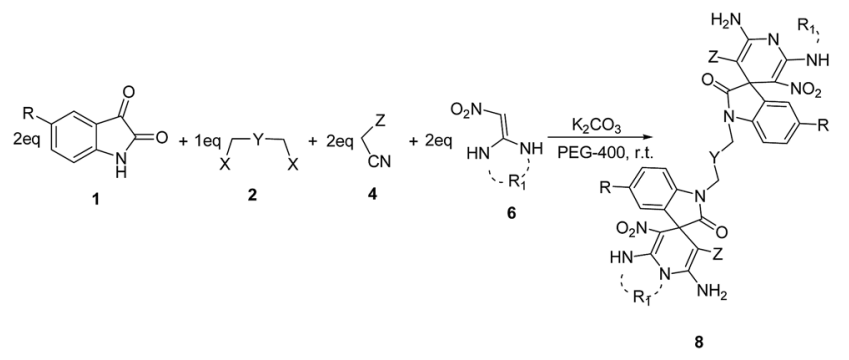

\begin{tabular}{|c|c|c|c|c|c|c|c|c|}
\hline Entry & $\mathrm{R}$ & $\mathrm{Y}$ & $\mathrm{X}$ & $\mathrm{Z}$ & Ketene aminal & Product & Time (h) & Yield $^{a}(\%)$ \\
\hline 1 & $\mathrm{H}$ & $\left(\mathrm{CH}_{2}\right)_{2}$ & $\mathrm{Br}$ & $\mathrm{CN}$ & $6 a$ & $8 a$ & 11 & 73 \\
\hline 2 & $\mathrm{H}$ & $\left(\mathrm{CH}_{2}\right)_{4}$ & $\mathrm{Br}$ & $\mathrm{CN}$ & $6 b$ & $8 b$ & 12 & 74 \\
\hline 3 & $\mathrm{H}$ & $\left(p-\mathrm{C}_{6} \mathrm{H}_{4}\right)$ & $\mathrm{Cl}$ & $\mathrm{CN}$ & $6 c$ & $8 c$ & 11 & 69 \\
\hline 4 & $\mathrm{NO}_{2}$ & $\left(\mathrm{CH}_{2}\right)_{2}$ & $\mathrm{Br}$ & $\mathrm{CN}$ & $6 b$ & 8d & 13 & 73 \\
\hline 5 & $\mathrm{NO}_{2}$ & $\left(\mathrm{CH}_{2}\right)_{4}$ & $\mathrm{Br}$ & $\mathrm{CN}$ & 6d & $8 e$ & 10 & 75 \\
\hline 6 & $\mathrm{NO}_{2}$ & $\left(p-\mathrm{C}_{6} \mathrm{H}_{4}\right)$ & $\mathrm{Cl}$ & $\mathrm{CN}$ & $6 a$ & $8 f$ & 10 & 70 \\
\hline 7 & $\mathrm{CH}_{3}$ & $\left(\mathrm{CH}_{2}\right)_{4}$ & $\mathrm{Br}$ & $\mathrm{CN}$ & $6 c$ & $8 g$ & 9 & 72 \\
\hline 8 & $\mathrm{CH}_{3}$ & $\left(o-\mathrm{C}_{6} \mathrm{H}_{4}\right)$ & $\mathrm{Br}$ & $\mathrm{CN}$ & $6 a$ & $8 h$ & 11 & 69 \\
\hline 9 & $\mathrm{CH}_{3}$ & $\left(p-\mathrm{C}_{6} \mathrm{H}_{4}\right)$ & $\mathrm{Cl}$ & $\mathrm{CN}$ & $6 b$ & $8 \mathbf{i}$ & 9 & 71 \\
\hline 10 & $\mathrm{~F}$ & $\left(\mathrm{CH}_{2}\right)_{2}$ & $\mathrm{Br}$ & $\mathrm{CN}$ & $8 b$ & $8 \mathbf{j}$ & 12 & 67 \\
\hline 11 & $\mathrm{~F}$ & $\left(p-\mathrm{C}_{6} \mathrm{H}_{4}\right)$ & $\mathrm{Cl}$ & $\mathrm{CN}$ & $6 d$ & $8 k$ & 10 & 69 \\
\hline 12 & $\mathrm{Br}$ & $\left(\mathrm{CH}_{2}\right)_{4}$ & $\mathrm{Br}$ & $\mathrm{CN}$ & $6 a$ & 81 & 12 & 72 \\
\hline 13 & $\mathrm{Br}$ & $\left(o-\mathrm{C}_{6} \mathrm{H}_{4}\right)$ & $\mathrm{Br}$ & $\mathrm{CN}$ & $6 c$ & $8 \mathrm{~m}$ & 13 & 71 \\
\hline 14 & $\mathrm{Br}$ & $\left(o-\mathrm{C}_{6} \mathrm{H}_{4}\right)$ & $\mathrm{Cl}$ & $\mathrm{CN}$ & 6d & $8 n$ & 10 & 77 \\
\hline
\end{tabular}

${ }^{a}$ Isolated yield. 
aminals were successfully utilized to prepare the corresponding bis-spiro-indoline-pyrido-pyrimidines, bis-spiro-indolineimidazo-pyridines and bis-spiro-indoline-pyridines derivatives in high yields. Noteworthy, as a result of the greater nucleophilicity of the $\alpha$-carbon atom in comparison with nitrogen atom, ${ }^{43}$ attack of $\alpha$-carbon leads to the reaction between ketene aminals (6a-6d) and bis-isatin-malononitrile aggregate to form the corresponding spiro-dihydropyridine ring in the target compounds (8a-8n) in moderate to good yields (67-75\%) (upper pathway of Scheme 2). Recent studies on mono-spiro counterparts of bis-spirooxindoles that contain dihydropyridine rings have approved their ability in inhibition of acetyl cholinesterase and butyryl cholinesterase enzymes which are well known for they role in Alzheimer disease that it makes these bis-spiro molecules an attractive subjects of further biological investigation. ${ }^{21}$

It should be mentioned that the reaction failed when ethyl-2cyanoacetate and methyl-2-cyanoacetate were used instead of malononitrile which could be related to lower tendency of ketene aminals to undergo condensation reactions when compared to enolizable ketones.

A plausible mechanism for the synthesis of bisspirooxindole derivatives 7 and 8 in the presence of $\mathrm{K}_{2} \mathrm{CO}_{3}$ is shown on Scheme 3. The first step involves the formation of product 3 from condensation reaction between 2 eq. of isatin 1 with 1 eq. dihalide 2 . In the PEG-400/ $\mathrm{K}_{2} \mathrm{CO}_{3}$ system, because of the coordination of the cationic centre of $\mathrm{K}_{2} \mathrm{CO}_{3}$ with the oxygen of polyethylene glycol, the $\mathrm{CO}_{3}{ }^{2-}$ anion could be presented in solution ${ }^{44}$ and the reaction of $\mathrm{CO}_{3}{ }^{2-}$ anion with isatin derivatives was elevated by increasing the nucleophilicity of nitrogen atom to attack to dihalide. Then, fast Knoevenagel condensation occurs between $\mathbf{3}$ and acidic protons of compound $\mathbf{4}$ for the formation of olefin A. In the presence of $\mathrm{K}_{2} \mathrm{CO}_{3}$, ketene aminal 6 converts to its corresponding enolate, which easily reacts with olefin $\mathbf{A}$ to produce intermediate $\mathbf{B}$, which followed by proton transfer and tautomerization to form the desired product $\mathbf{8}$.

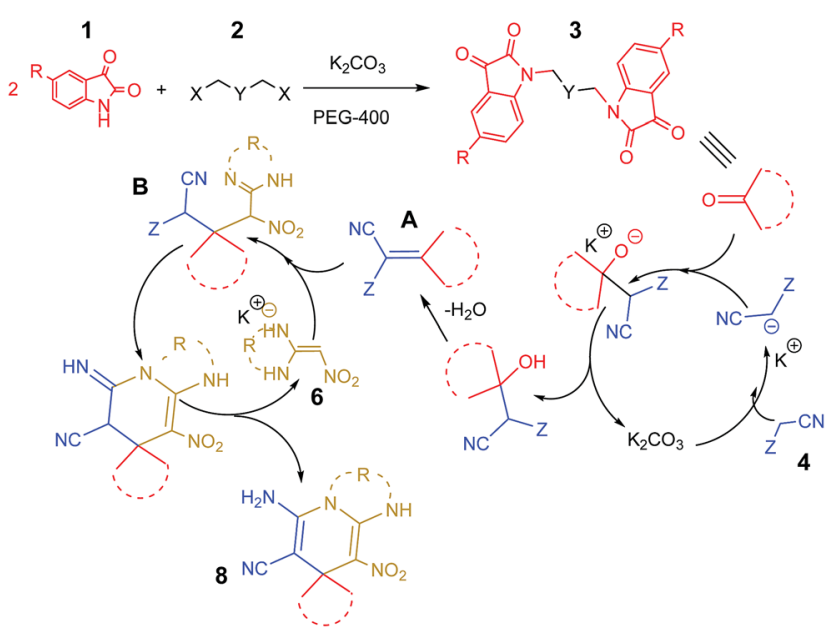

Scheme 3 The proposed mechanism for the synthesis of bis-spirooxindole in the presence of $\mathrm{K}_{2} \mathrm{CO}_{3}$ in PEG-400.

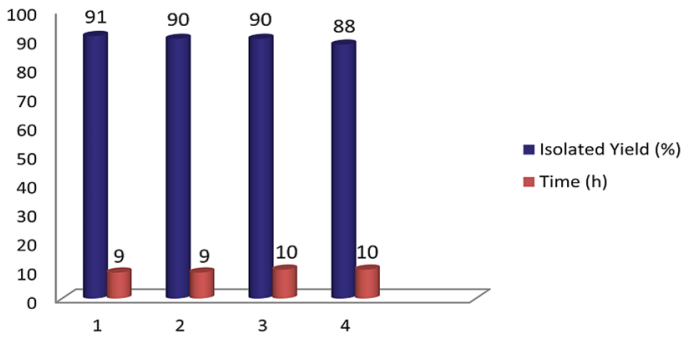

Chart 1 The reaction of: isatin $1 \mathrm{a}(2 \mathrm{mmol})$ with 1,6 -dibromohexane 2a (1 mmol), malononitrile $4 \mathrm{a}(2 \mathrm{mmol})$ and dimedone $5 \mathrm{a}(2 \mathrm{mmol})$ in the presence of recovered PEG-400/ $\mathrm{K}_{2} \mathrm{CO}_{3}(2 \mathrm{mmol})$ at room temperature.

In another study, reusability of PEG and $\mathrm{K}_{2} \mathrm{CO}_{3}$ were studied. For this propose, the reaction of isatin $1 \mathrm{a}(2 \mathrm{mmol})$ with 1,6dibromohexane $2 \mathbf{a}(1 \mathrm{mmol})$, malononitrile $4 \mathbf{4}(2 \mathrm{mmol})$ and dimedone $5 \mathbf{a}(2 \mathrm{mmol})$ was performed in the presence of $\mathrm{K}_{2} \mathrm{CO}_{3}$ (2 mmol) in PEG-400 (3 mL) at room temperature. After completion of the reaction, as monitored by TLC, water $(3 \mathrm{~mL})$ was added to the reaction mixture to precipitate the product and separate it. After isolation of product, water was removed by direct distillation at $100{ }^{\circ} \mathrm{C}$ and the mixture of PEG-400 and $\mathrm{K}_{2} \mathrm{CO}_{3}$ was washed with diethyl ether (two times, every time with $2 \mathrm{~mL}$ ). The regenerated PEG and $\mathrm{K}_{2} \mathrm{CO}_{3}$ were successfully used for the next run under identical reaction conditions. The reusability results are summarized in Chart 1 . As Chart 1 indicates, $\mathrm{PEG} / \mathrm{K}_{2} \mathrm{CO}_{3}$ system was successfully recycled and reused in four cycles with almost unchanged yields (Chart 1 ).

\section{Experimental}

All reagents were purchased from commercial suppliers and used without any further purification. All the reactions were carried out under air atmosphere. $\mathrm{H}$ and $\mathrm{C}$ NMR spectra were recorded on a Bruker Avance (400 and $100 \mathrm{MHz}$ ) NMR spectrometer in DMSO as solvent. The following abbreviations are used to indicate the multiplicity in NMR spectra: $\mathrm{s}$ - singlet; $\mathrm{d}$ doublet; t-triplet; q-quartet; quint.-quintet; dd-double doublet; $\mathrm{m}$-multiplet; br-broad signal and coupling constants ( $J$ values) in hertz $(\mathrm{Hz})$. Melting points were recorded on a Büchi B-545 apparatus in open capillary tubes. Elemental analyses were performed by using a Leco Analyzer 932 (Leco Corp., St. Joseph, MI).

\section{General procedure for the synthesis of ketene aminals}

A solution of bis(methylthio)-2-nitroethylene $(1 \mathrm{mmol})$ and diamine derivatives $(1 \mathrm{mmol})$ or benzyl amine $(2 \mathrm{mmol})$ were added in a $25 \mathrm{~mL}$ round-bottomed flask contained ethanol $(4.0 \mathrm{~mL})$, and the resulting mixture was reflux at $80{ }^{\circ} \mathrm{C}$. After completion of the reaction confirmed by TLC, the reaction mixture was cooled to room temperature. Then, the precipitated product was filtered, and washed with cool ethanol (two times, every time with $4 \mathrm{~mL}$ ) to give the pure product. 
General procedure for the synthesis of bis-spirooxindole derivatives

A solution of isatin derivates $(2 \mathrm{mmol})$ and $\mathrm{K}_{2} \mathrm{CO}_{3}(276 \mathrm{mg}$, $2 \mathrm{mmol}$ ) in PEG (3 $\mathrm{mL})$ was stirred at room temperature. After $2 \mathrm{~h}$, di-halide derivates $(1 \mathrm{mmol})$ was added, and the mixture was stirred for $6 \mathrm{~h}$ at room temperature. After completion of the reaction confirmed by TLC, malono derivative $(2 \mathrm{mmol})$ and enolizable carbonyl compound (5a-5d, $2 \mathrm{mmol}$ ) or ketene aminal derivative $(\mathbf{6 a}-\mathbf{6} \mathbf{d}, 2 \mathrm{mmol})$ were added to the reaction mixture. After completion of the reaction, $3 \mathrm{~mL}$ of water was added to the reaction mixture; the precipitate was filtrated and washed with ethanol (two times, every time with $5 \mathrm{~mL}$ ) to give the pure product. The recovered PEG-400 and $\mathrm{K}_{2} \mathrm{CO}_{3}$ were used for another time without loss of its activity.

$1^{\prime}, \mathbf{1}^{\prime \prime \prime}$-(Hexane-1,6-diyl)bis(2-amino-7,7-dimethyl-2',5-dioxo5,6,7,8-tetrahydrospiro[chromene-4, $3^{\prime}$-indoline]-3-carbonitrile) (7a). Cream solid; mp $>290{ }^{\circ} \mathrm{C} ;{ }^{1} \mathrm{H}$ NMR (400 MHz, DMSO- $d_{6}$ ): $\delta(\mathrm{ppm})=1.02(\mathrm{~s}, 6 \mathrm{H}), 1.04(\mathrm{~s}, 6 \mathrm{H}), 1.41-1.44(\mathrm{~m}, 4 \mathrm{H}), 1.59-1.63$ (m, 4H), 2.09-2.19 (m, 4H), $3.04(\mathrm{~s}, 4 \mathrm{H}), 3.59-3.67$ (m, 4H), 6.86$6.88(\mathrm{~m}, 4 \mathrm{H}), 7.02-7.04(\mathrm{~m}, 2 \mathrm{H}), 7.25$ (br, 4H, $\left.\mathrm{NH}_{2}\right) ;{ }^{13} \mathrm{C} \mathrm{NMR}$ $\left(100 \mathrm{MHz}, \mathrm{DMSO}-d_{6}\right): \delta=26.5,27.5,28.1,32.4,45.2,46.8,50.4$, $57.7,108.8,111.2,117.7,122.6,123.4,128.8,134.2$, 143.5, 159.3, 164.7, 176.8, 195.3.

Dimethyl-1' , $^{\prime \prime \prime}$-(hexane-1,6-diyl)bis(2-amino-7,7-dimethyl2',5-dioxo-5,6,7,8-tetrahydrospiro[chromene-4,3'-indoline]-3carboxylate) (7b). Cream solid; $\mathrm{mp}>290{ }^{\circ} \mathrm{C} ;{ }^{1} \mathrm{H} \mathrm{NMR}(400 \mathrm{MHz}$, DMSO- $\left.d_{6}\right): \delta(\mathrm{ppm})=0.94(\mathrm{~s}, 6 \mathrm{H}), 1.01(\mathrm{~s}, 6 \mathrm{H}), 1.26(\mathrm{~s}, 4 \mathrm{H}), 1.50-$ $1.54(\mathrm{~m}, 4 \mathrm{H}), 1.66-1.68(\mathrm{~m}, 4 \mathrm{H}), 1.97-2.01$ (m, 2H), 2.13-2.17 (m, 2H), $3.21(\mathrm{~s}, 6 \mathrm{H}), 3.61-3.63(\mathrm{~m}, 4 \mathrm{H}), 6.86-6.90(\mathrm{~m}, 6 \mathrm{H}), 7.13$ (s, 2H), 7.87 (br, $\left.4 \mathrm{H}, \mathrm{NH}_{2}\right) ;{ }^{13} \mathrm{C}$ NMR (100 MHz, DMSO- $\left.d_{6}\right): \delta=$ 20.0, 27.5, 31.2, 37.4, 44.3, 47.0, 59.2, 76.2, 108.7, 109.9, 113.7, $125.7,128.2$, 130.3, 136.0, 138.3, 144.7, 150.2, 159.7, 165.8, 167.7, 178.7, 196.4 .

Diethyl-1' $\mathbf{1}^{\prime \prime \prime}$-(hexane-1,6-diyl)bis(2-amino-7,7-dimethyl-2' ${ }^{\prime}$,5dioxo-5,6,7,8-tetrahydrospiro[chromene-4, $3^{\prime}$-indoline]-3-carboxylate) (7c). Cream solid; $\mathrm{mp}>290{ }^{\circ} \mathrm{C} ;{ }^{1} \mathrm{H}$ NMR $(400 \mathrm{MHz}$, DMSO- $\left.d_{6}\right): \delta=0.68-0.72(\mathrm{~m}, 6 \mathrm{H}), 0.93-0.97(\mathrm{~s}, 6 \mathrm{H}), 1.02(\mathrm{~s}, 6 \mathrm{H})$, $1.26(\mathrm{~s}, 4 \mathrm{H}), 1.50-1.52(\mathrm{~m}, 4 \mathrm{H}), 1.67-1.69(\mathrm{~m}, 4 \mathrm{H}), 2.02-2.13(\mathrm{~m}$, $4 \mathrm{H}), 3.35-3.67(\mathrm{~m}, 4 \mathrm{H}), 3.71-3.73(\mathrm{~m}, 4 \mathrm{H}), 6.86-6.91(\mathrm{~m}, 6 \mathrm{H})$, 7.14 (s, 2H), 7.92 (br, $\left.4 \mathrm{H}, \mathrm{NH}_{2}\right) ;{ }^{13} \mathrm{C}$ NMR (100 MHz, DMSO- $\left.d_{6}\right)$ : $\delta=14.0,19.6,26.7,27.2,27.4,31.2,44.6,46.5,50.9,53.4,59.3$, 75.9, 101.2, 107.7, 128.4, 134.3, 145.4, 158.4, 159.4, 159.5, 159.9, 163.7, 167.8, 177.5.

Dimethyl-1,1" ${ }^{\prime \prime}$-(hexane-1,6-diyl)bis ( $2^{\prime}$-amino-6' -(hydroxymethyl)-5-methyl-2,8' -dioxo-8 $^{\prime} \boldsymbol{H}$-spiro[indoline-3, $\mathbf{4}^{\prime}$-pyrano[3,2b]pyran]-3'-carboxylate) (7d). Cream solid; mp $>290{ }^{\circ} \mathrm{C} ;{ }^{1} \mathrm{H}$ NMR (400 MHz, DMSO- $\left.d_{6}\right): \delta=1.44-1.48(\mathrm{~m}, 4 \mathrm{H}), 1.64-1.66(\mathrm{~m}, 4 \mathrm{H})$, $2.23(\mathrm{~s}, 6 \mathrm{H}), 3.25(\mathrm{~s} 6 \mathrm{H}), 3.67-3.73(\mathrm{~m}, 4 \mathrm{H}), 3.93-4.06(\mathrm{~m}, 4 \mathrm{H})$, 5.64 (br, 2H, OH), 6.33 (s, 2H), 6.96-7.01 (m, 4H), 7.07-7.09 (m, $2 \mathrm{H}), 8.11$ (br, $\left.4 \mathrm{H}, \mathrm{NH}_{2}\right) ;{ }^{13} \mathrm{C}$ NMR $\left(100 \mathrm{MHz}, \mathrm{DMSO}-d_{6}\right): \delta=26.2$, 27.2, 31.2, 45.1, 51.1, 59.4, 73.9, 73.9, 108.7, 111.8, 124.4, 129.8, $131.9,133.5$, 136.8, 141.4, 148.3, 160.4, 167.5, 168.8, 169.8, 175.7 .

Diethyl-1,1" ${ }^{\prime \prime}$-(hexane-1,6-diyl)bis (2'-amino-5, $7^{\prime}$-dimethyl-2, $5^{\prime}$ dioxo-5 ${ }^{\prime} H$-spiro[indoline-3,4'-pyrano $[4,3-b]$ pyran $]-3^{\prime}$-carboxylate) (7e). Cream solid; mp $>290{ }^{\circ} \mathrm{C} ;{ }^{1} \mathrm{H}$ NMR (400 MHz, DMSO- $\left.d_{6}\right)$ : $\delta=0.73(\mathrm{t}, J=8 \mathrm{~Hz}, 6 \mathrm{H}), 1.46-1.47(\mathrm{~m}, 4 \mathrm{H}), 1.63-1.65(\mathrm{~m}, 4 \mathrm{H})$, $2.20(\mathrm{~s}, 6 \mathrm{H}), 2.21(\mathrm{~s}, 6 \mathrm{H}) 3.45-3.52(\mathrm{~m}, 2 \mathrm{H}), 3.63-3.68(\mathrm{~m}, 2 \mathrm{H})$, 3.70-3.75 (m, 4H), $6.31(\mathrm{~s}, 2 \mathrm{H}), 6.78-6.82(\mathrm{~m}, 4 \mathrm{H}), 6.98(\mathrm{~d}, J=$ $8 \mathrm{~Hz}, 2 \mathrm{H}), 8.07$ (br, $\left.4 \mathrm{H}, \mathrm{NH}_{2}\right) ;{ }^{13} \mathrm{C}$ NMR $\left(100 \mathrm{MHz}, \mathrm{DMSO}-d_{6}\right): \delta=$ 14.0, 19.6, 21.0, 26.8, 27.2, 44.6, 46.6, 59.3, 75.9, 98.1, 101.3, $107.4,123.9,128.5,130.7,134.5,143.1,159.4,159.5,159.9,163.6$, 167.7, 177.4 .

Diethyl-1' ${ }^{\prime} 1^{\prime \prime \prime}$-(hexane-1,6-diyl)bis(2-amino-5'-bromo-2' ${ }^{\prime}, 5$ dioxo-5,6,7,8-tetrahydrospiro[chromene-4, $3^{\prime}$-indoline]-3-carboxylate) (7f). Cream solid; $\mathrm{mp}>290{ }^{\circ} \mathrm{C} ;{ }^{1} \mathrm{H}$ NMR $(400 \mathrm{MHz}$, DMSO- $\left.d_{6}\right): \delta=0.71-0.74(\mathrm{~m}, 6 \mathrm{H}), 1.47-1.49(\mathrm{~m}, 4 \mathrm{H}), 1.64-1.66$ $(\mathrm{m}, 4 \mathrm{H}), 1.88-1.89(\mathrm{~m}, 4 \mathrm{H}), 2.17-2.22(\mathrm{~m}, 4 \mathrm{H}), 2.64-2.67$ (m, $4 \mathrm{H}), 3.47-3.50(\mathrm{~m}, 2 \mathrm{H}), 3.62-3.65(\mathrm{~m}, 2 \mathrm{H}), 3.67-3.77(\mathrm{~m}, 4 \mathrm{H})$, 6.84-6.87 (m, 2H), 7.12-7.13 (m, 2H), 7.29-7.33 (m, 2H), 7.99 (br, $\left.4 \mathrm{H}, \mathrm{NH}_{2}\right) ;{ }^{13} \mathrm{C}$ NMR (100 MHz, DMSO- $\left.d_{6}\right): \delta=14.1,20.0$, 26.7, 27.0, 27.4, 37.4, 44.6, 46.84, 59.2, 76.0, 109.3, 113.2, 113.9, 125.7, 130.4, 138.3, 144.8, 159.7, 165.4, 167.8, 178.1, 195.5.

$\mathbf{1}^{\prime}, \mathbf{1}^{\prime \prime \prime}$-(Hexane-1,6-diyl)bis(2-amino-5' -bromo-7,7-dimethyl$2^{\prime}, 5$-dioxo-5,6,7,8-tetrahydrospiro[chromene-4, $3^{\prime}$-indoline]-3carbonitrile) (7g). White solid, $\mathrm{mp}>290{ }^{\circ} \mathrm{C} ;{ }^{1} \mathrm{H}$ NMR $(400 \mathrm{MHz}$, DMSO- $\left.d_{6}\right): \delta=1.02(\mathrm{~s}, 6 \mathrm{H}), 1.07(\mathrm{~s}, 6 \mathrm{H}), 1.40-1.42(\mathrm{~m}, 4 \mathrm{H}), 1.58-$ $1.60(\mathrm{~m}, 4 \mathrm{H}), 2.13-1.16(\mathrm{~m}, 4 \mathrm{H}), 2.59(\mathrm{~s}, 4 \mathrm{H}), 2.64(\mathrm{~s}, 4 \mathrm{H}), 3.57-$ $3.71(\mathrm{~m}, 4 \mathrm{H}), 6.97-6.99(\mathrm{~m}, 2 \mathrm{H}), 7.29-7.30(\mathrm{~m}, 2 \mathrm{H}), 7.39$ (br, $4 \mathrm{H}$, $\left.\mathrm{NH}_{2}\right) ;{ }^{13} \mathrm{C}$ NMR (100 MHz, DMSO- $\left.d_{6}\right): \delta=26.3,27.1,27.7,28.0$, 32.5 , 47.0, 50.0, 57.0, 61.5, 110.6, 110.8, 114.4, 118.9, 126.3, 131.5, 136.6, 142.9, 148.3, 155.2, 159.4, 165.2, 176.5, 195.6.

Dimethyl-1,1" $1^{\prime \prime}$-(hexane-1,6-diyl)bis( $2^{\prime}$-amino-5, $7^{\prime}$-dimethyl2,5'-dioxo-5' $H$-spiro[indoline-3, $\mathbf{4}^{\prime}$-pyrano[4,3-b]pyran]-3'-carboxylate) (7h). Light brown solid, mp $>290{ }^{\circ} \mathrm{C}$; ${ }^{1} \mathrm{H}$ NMR $(400 \mathrm{MHz}$, DMSO- $\left.d_{6}\right): \delta=1.47-1.49(\mathrm{~m}, 4 \mathrm{H}), 1.61-1.64(\mathrm{~m}, 4 \mathrm{H}), 2.20(\mathrm{~s}$, $6 \mathrm{H}), 2.21(\mathrm{~s}, 6 \mathrm{H}), 3.24(\mathrm{~s}, 6 \mathrm{H}), 3.60-3.72(\mathrm{~m}, 4 \mathrm{H}), 6.31(\mathrm{~s}, 2 \mathrm{H})$, 6.79-6.81 (m, 4H), 6.96-98 (m, 2H), $8.02\left(\mathrm{br}, 4 \mathrm{H}, \mathrm{NH}_{2}\right) ;{ }^{13} \mathrm{C} \mathrm{NMR}$ (100 MHz, DMSO- $d_{6}$ ): $\delta=19.6,21.0,26.7,27.2$, 27.4, 44.5, 46.6, 50.9, 59.3, 76.0, 101.3, 107.5, 123.9, 128.6, 130.7, 134.3, 142.9, 158.8, 159.4, 163.6, 167.859, 177.4 .

Dimethyl-1,1" -(hexane-1,6-diyl)bis( $2^{\prime}$-amino-5-fluoro- $7^{\prime}$ methyl-2, $5^{\prime}$-dioxo- $5^{\prime} H$-spiro[indoline-3, $4^{\prime}$-pyrano[4,3-b]pyran]$3^{\prime}$-carboxylate) (7i). White solid, $\mathrm{mp}>290{ }^{\circ} \mathrm{C} ;{ }^{1} \mathrm{H}$ NMR (400 MHz, DMSO- $\left.d_{6}\right): \delta=1.48-1.50(\mathrm{~m}, 4 \mathrm{H}), 1.64-1.66(\mathrm{~m}, 4 \mathrm{H}), 2.21$ $(\mathrm{s}, 6 \mathrm{H}), 3.23(\mathrm{~s}, 6 \mathrm{H}), 3.52-3.74(\mathrm{~m}, 4 \mathrm{H}), 6.32(\mathrm{~s}, 2 \mathrm{H}), 6.86-6.93$ $(\mathrm{m}, 2 \mathrm{H}), 6.99-7.01(\mathrm{~m}, 2 \mathrm{H}), 7.16-7.20(\mathrm{~m}, 2 \mathrm{H}), 8.03(\mathrm{br}, 4 \mathrm{H}$, $\left.\mathrm{NH}_{2}\right) ;{ }^{13} \mathrm{C}$ NMR (100 MHz, DMSO- $\left.d_{6}\right): \delta=19.9,27.0,27.4,27.5$, 44.6, 46.4, 51.1, 59.1, 75.9, 101.4, 107.9, 124.1, 128.6, 130.7, 134.5, 143.1, 158.6, 159.4, 163.6, 167.9, 177.5.

Dimethyl-1,1' ${ }^{\prime \prime}$-(butane-1,4-diyl)bis (2'-amino-2, $5^{\prime}$-dioxo-5' $\mathbf{H}$ spiro[indoline-3, $\mathbf{4}^{\prime}$-pyrano[3,2-c] chromene]-3'-carboxylate) (7j). Light brown solid, mp $>290{ }^{\circ} \mathrm{C},{ }^{1} \mathrm{H}$ NMR (400 MHz, DMSO- $\left.d_{6}\right)$ : $\delta=1.77-1.85(\mathrm{~m}, 4 \mathrm{H}), 3.05(\mathrm{~s}, 6 \mathrm{H}), 3.66-3.83(\mathrm{~m}, 4 \mathrm{H}), 6.82-6.90$ $(\mathrm{m}, 2 \mathrm{H}), 7.02-7.25(\mathrm{~m}, 6 \mathrm{H}), 7.48(\mathrm{~d}, J=8 \mathrm{~Hz}, 2 \mathrm{H}), 7.54-7.57(\mathrm{~m}$, $2 \mathrm{H}), 7.75-7.84(\mathrm{~m}, 2 \mathrm{H}), 8.07$ (d, $J=8 \mathrm{~Hz}, 2 \mathrm{H}), 8.21$ (br, $4 \mathrm{H}, \mathrm{NH}_{2}$ ); ${ }^{13} \mathrm{C}$ NMR (100 MHz, DMSO- $\left.d_{6}\right): \delta=31.2,49.0,58.3,113.0,116.0$, $117.3,121.3,123.4,125.7,126.9$, 127.9, 128.2, 130.7, 134.5, 135.4, 137.1, 137.4, 147.4, 152.6, 155.4, 158.5, 159.1, 162.4.

$1^{\prime}, \mathbf{1}^{\prime \prime \prime}$-(Hexane-1,6-diyl)bis(2-amino-5' $, 7,7$-trimethyl-2' ${ }^{\prime}, 5$ dioxo-5,6,7,8-tetrahydrospiro[chromene-4, $3^{\prime}$-indoline]-3-carbonitrile) (7k). Cream solid, $\mathrm{mp}>290{ }^{\circ} \mathrm{C} ;{ }^{1} \mathrm{H}$ NMR $(400 \mathrm{MHz}$, DMSO- $\left.d_{6}\right): \delta=1.02(\mathrm{~s}, 6 \mathrm{H}), 1.04(\mathrm{~s}, 6 \mathrm{H}), 1.41-1.44(\mathrm{~m}, 4 \mathrm{H}), 1.59-$ 
$1.63(\mathrm{~m}, 4 \mathrm{H}), 2.09-2.19(\mathrm{~m}, 4 \mathrm{H}), 2.25(\mathrm{~s}, 6 \mathrm{H}), 3.04(\mathrm{~s}, 4 \mathrm{H}), 3.59-$ $3.67(\mathrm{~m}, 4 \mathrm{H}), 6.86-6.88(\mathrm{~m}, 4 \mathrm{H}), 7.02-7.05(\mathrm{~m}, 2 \mathrm{H}), 7.45(\mathrm{br}, 4 \mathrm{H}$, $\left.\mathrm{NH}_{2}\right) ;{ }^{13} \mathrm{C}$ NMR (100 MHz, DMSO- $\left.d_{6}\right): \delta=21.1,26.5,27.2,27.7$, 27.9, 32.4, 44.5, 46.9, 50.4, 57.9, 108.5, 111.3, 117.7, 124.0, 129.0, 131.5, 134.3, 141.1, 159.2, 159.3, 164.6, 176.7, 195.3.

Dimethyl-1' , $^{\prime \prime \prime}$-(hexane-1,6-diyl)bis(2-amino-2' ${ }^{\prime}$, -dioxo5,6,7,8-tetrahydrospiro[chromene-4, $\mathbf{3}^{\prime}$-indoline]-3-carboxylate) (7l). Cream solid, $\mathrm{mp}>290{ }^{\circ} \mathrm{C} ;{ }^{1} \mathrm{H}$ NMR (400 MHz, DMSO- $d_{6}$ ): $\delta=1.50-1.52(\mathrm{~m}, 4 \mathrm{H}), 1.66-1.68(\mathrm{~m}, 4 \mathrm{H}), 1.86-1.88(\mathrm{~m}, 4 \mathrm{H}), 2.11$ (s, 6H), 2.64-2.66 (m, 4H), 3.19-3.21 (m, 4H), 3.62-3.71 (m, 4H), 6.82-6.85 (m, 4H), 6.91-6.93 (d, $J=7 \mathrm{~Hz}, 2 \mathrm{H}), 7.11-7.14(\mathrm{~m}, 2 \mathrm{H})$, $7.86\left(\mathrm{r}, 4 \mathrm{H}, \mathrm{NH}_{2}\right) ;{ }^{13} \mathrm{C}$ NMR (100 MHz, DMSO- $\left.d_{6}\right): \delta=14.1,20.1$, 26.8, 27.4, 31.2, 37.4, 46.7, 50.7, 76.6, 107.3, 114.6, 121.5, 122.8, 127.9, 135.6, 145.2, 159.5, 164.9, 168.1, 178.5, 195.3.

Diethyl-1,1" -(hexane-1,6-diyl)bis(2' -amino-6' -(hydroxymethyl)2,8'-dioxo-8' $H$-spiro[indoline-3, $\mathbf{4}^{\prime}$-pyrano[3,2-b]pyran]-3' -carboxylate) (7m). Light brown solid, $\mathrm{mp}>290{ }^{\circ} \mathrm{C} ;{ }^{1} \mathrm{H}$ NMR $(400 \mathrm{MHz}$, DMSO- $\left.d_{6}\right): \delta=0.68-0.72(\mathrm{~m}, 6 \mathrm{H}), 1.43-1.45(\mathrm{~m}, 4 \mathrm{H}), 1.64-1.66$ $(\mathrm{m}, 4 \mathrm{H}), 3.57-3.70(\mathrm{~m}, 4 \mathrm{H}), 3.71-3.85(\mathrm{~m}, 4 \mathrm{H}), 3.97-3.98(\mathrm{~m}$, $4 \mathrm{H}), 5.63(\mathrm{t}, J=6.4 \mathrm{~Hz}, 2 \mathrm{H}, \mathrm{OH}), 6.35(\mathrm{~s}, 2 \mathrm{H}), 7.08-7.10(\mathrm{~m}, 2 \mathrm{H})$, 7.49-7.50 (m, 4H), 8.20 (br, 4H, $\left.\mathrm{NH}_{2}\right) ;{ }^{13} \mathrm{C}$ NMR $(100 \mathrm{MHz}$, DMSO- $\left.d_{6}\right): \delta=14.0,26.2,27.2,44.0,51.2,59.4,73.3,80.0,110.0$, $111.9,114.7,126.9,132.2,135.7,137.1,143.2$, 147.2, 160.6, 167.2, 168.8, 169.9, 175.7 .

Diethyl-1,1' dioxo-5' $H$-spiro[indoline-3, $4^{\prime}$-pyrano $[4,3-b]$ pyran $]-3^{\prime}$-carboxylate) (7n). Cream solid, mp $>290{ }^{\circ} \mathrm{C} ;{ }^{1} \mathrm{H}$ NMR (400 MHz, DMSO$\left.d_{6}\right): \delta=0.63-0.68(\mathrm{~m}, 6 \mathrm{H}), 1.80(\mathrm{~m}, 4 \mathrm{H}), 2.23(\mathrm{~s}, 6 \mathrm{H}), 3.59-3.66$ $(\mathrm{m}, 4 \mathrm{H}) 3.73-3.80(\mathrm{~m}, 4 \mathrm{H}), 6.34(\mathrm{~s}, 2 \mathrm{H}), 6.86-6.90(\mathrm{~m}, 2 \mathrm{H}), 6.98-$ $7.02(\mathrm{~m}, 4 \mathrm{H}), 7.14-7.20(\mathrm{~m}, 2 \mathrm{H}), 8.07$ (br, 4H, $\left.\mathrm{NH}_{2}\right) ;{ }^{13} \mathrm{C} \mathrm{NMR}$ (100 MHz, DMSO- $\left.d_{6}\right): \delta=14.0,19.7,25.2,46.6,59.2,75.8,98.2$, 101.2 , 107.9, 121.9, 123.2, 128.3, 134.6, 145.4, 158.8, 159.5, 160.0, 163.7, 167.7, 177.7 .

Dimethyl-1' , $^{\prime \prime \prime}$-(butane-1,4-diyl)bis(2-amino-2', 5-dioxo5,6,7,8-tetrahydrospiro[chromene-4, $\mathbf{3}^{\prime}$-indoline]-3-carboxylate) (7o). White solid, $\mathrm{mp}>290{ }^{\circ} \mathrm{C}$; ${ }^{1} \mathrm{H}$ NMR (400 MHz, DMSO- $d_{6}$ ): $\delta=1.78-1.80(\mathrm{~m}, 4 \mathrm{H}), 1.87-1.89(\mathrm{~m}, 4 \mathrm{H}), 2.15-2.24(\mathrm{~m}, 4 \mathrm{H})$, 2.66-2.68 (m, 4H), $3.19(\mathrm{~s}, 6 \mathrm{H}), 3.70-3.75(\mathrm{~m}, 4 \mathrm{H}), 6.81-6.86(\mathrm{~m}$, 2H), 6.91-6.95 (m, 4H), 7.09-7.14 (m, 2H), 7.89 (br, 4H, $\mathrm{NH}_{2}$ ); ${ }^{13} \mathrm{C}$ NMR (100 MHz, DMSO- $\left.d_{6}\right): \delta=20.2,26.1,28.3,38.0,43.2$, 45.3, 59.2, 75.8, 107.1, 113.1, 114.0, 124.9, 130.4, 138.3, 145.1, 159.7, 165.3, 168.2, 178.2, 194.9.

$1^{\prime}, 1^{\prime \prime \prime}$-(1,4-Phenylenebis(methylene))bis(2-amino-5'-bromo7,7-dimethyl-2' ,5-dioxo-5,6,7,8-tetrahydrospiro[chromene-4,3'indoline]-3-carbonitrile) (7p). Cream solid, $\mathrm{mp}>290{ }^{\circ} \mathrm{C} ;{ }^{1} \mathrm{H}$ NMR (400 MHz, DMSO- $\left.d_{6}\right): \delta=1.04(\mathrm{~s}, 6 \mathrm{H}), 1.06(\mathrm{~s}, 6 \mathrm{H}), 2.21(\mathrm{~s}$, $4 \mathrm{H}), 2.60-2.68(\mathrm{~m}, 4 \mathrm{H}), 4.91(\mathrm{~s}, 4 \mathrm{H}), 6.67-6.71(\mathrm{~m}, 2 \mathrm{H}), 7.34-7.36$ (m, 4H), $7.43(\mathrm{~s}, 4 \mathrm{H}), 7.46\left(\mathrm{br}, 4 \mathrm{H}, \mathrm{NH}_{2}\right) ;{ }^{13} \mathrm{C} \mathrm{NMR}(100 \mathrm{MHz}$, DMSO- $\left.d_{6}\right): \delta=27.5,27.7,28.0,31.2,32.6,43.6,47.2,50.3,56.9$, $110.5,111.4,114.9,117.8,126.4,127.6,131.5,135.2,136.4$, 142.4, 159.5, 165.5, 176.8, 195.8 .

Diethyl-1' $\mathbf{1}^{\prime \prime \prime}$-(1,4-phenylenebis(methylene))bis(2-amino-5' bromo-2' ,5-dioxo-5,6,7,8-tetrahydrospiro[chromene-4, $3^{\prime}$-indoline]3-carboxylate) (7q). Cream solid, mp $>290{ }^{\circ} \mathrm{C}$; ${ }^{1} \mathrm{H}$ NMR $(400 \mathrm{MHz}$, DMSO- $\left.d_{6}\right): \delta=0.54-0.62(\mathrm{~m}, 6 \mathrm{H}), 1.91-1.94(\mathrm{~m}, 4 \mathrm{H}), 2.21-2.35(\mathrm{~m}$, $4 \mathrm{H}), 2.68-2.70(\mathrm{~m}, 4 \mathrm{H}), 3.42-4.49(\mathrm{~m}, 2 \mathrm{H}), 3.77-3.83(\mathrm{~m}, 2 \mathrm{H}), 4.70$ $(\mathrm{d}, J=16 \mathrm{~Hz}, 2 \mathrm{H}), 4.97(\mathrm{~d}, J=16 \mathrm{~Hz}, 2 \mathrm{H}), 6.63-6.65(\mathrm{~m}, 2 \mathrm{H}), 7.17-$
7.18 (m, 2H), 7.25-7.28 (m, 2H), 7.56 (s, 4H), 8.03 (br, 4H, $\mathrm{NH}_{2}$ ); ${ }^{13} \mathrm{C}$ NMR (100 MHz, DMSO- $\left.d_{6}\right): \delta=13.9,20.0,28.9,35.9,42.1$, 50.8, 51.1, 60.8, 81.1, 109.7, 11.7, 124.4, 129.9, 130.9, 133.5, 136.9, 141.4, 148.3, 160.4, 167.5, 168.9, 169.8, 175.9 .

$1^{\prime}, 1^{\prime \prime \prime}$-(1,4-Phenylenebis(methylene))bis(2-amino-5' -bromo$2^{\prime}, 5$-dioxo-5,6,7,8-tetrahydrospiro[chromene-4, $3^{\prime}$-indoline]-3carbonitrile) (7r). Cream solid, $\mathrm{mp}>290{ }^{\circ} \mathrm{C} ;{ }^{1} \mathrm{H} \mathrm{NMR}(400 \mathrm{MHz}$, DMSO- $\left.d_{6}\right): \delta=1.95-1.98(\mathrm{~m}, 4 \mathrm{H}), 2.25-2.29(\mathrm{~m}, 4 \mathrm{H}), 2.70-2.85$ $(\mathrm{m}, 4 \mathrm{H}), 4.90(\mathrm{~s}, 4 \mathrm{H}), 6.69-6.71(\mathrm{~m}, 2 \mathrm{H}), 6.97-6.99(\mathrm{~m}, 2 \mathrm{H}), 7.11-$ $7.14(\mathrm{~m}, 4 \mathrm{H}), 7.32(\mathrm{~s}, 2 \mathrm{H}), 7.45\left(\mathrm{br}, 4 \mathrm{H}, \mathrm{NH}_{2}\right) ;{ }^{13} \mathrm{C}$ NMR $(100$ MHz, DMSO- $\left.d_{6}\right): \delta=20.1,27.3,36.7,43.5,44.6,47.3,57.0$, 111.3 , 111.5, 114.9, 117.8, 126.6, 127.6, 131.5, 135.2, 136.6, 142.3, 159.2, 159.3, 167.5, 176.9, 195.9 .

Diethyl-1' $\mathbf{1}^{\prime \prime \prime}$-(1,4-phenylenebis(methylene))bis(2-amino$5^{\prime}, 7,7$-trimethyl-2' ,5-dioxo-5,6,7,8-tetrahydrospiro[chromene4,3'-indoline]-3-carboxylate) (7s). Cream solid, $\mathrm{mp}>290{ }^{\circ} \mathrm{C} ;{ }^{1} \mathrm{H}$ NMR (400 MHz, DMSO- $\left.d_{6}\right): \delta=0.53-0.61(\mathrm{~m}, 6 \mathrm{H}), 0.99(\mathrm{~s}, 6 \mathrm{H})$, $1.04(\mathrm{~s}, 6 \mathrm{H}), 1.26(\mathrm{~s}, 4 \mathrm{H}), 2.11(\mathrm{~s}, 4 \mathrm{H}), 2.17(\mathrm{~s}, 6 \mathrm{H}), 3.47-3.51(\mathrm{~m}$, $2 \mathrm{H}), 3.75-3.79(\mathrm{~m}, 2 \mathrm{H}), 4.66(\mathrm{~d}, J=16 \mathrm{~Hz}, 2 \mathrm{H}), 4.97(\mathrm{~d}, J=16 \mathrm{~Hz}$, $2 \mathrm{H}), 6.52-6.54(\mathrm{~m}, 2 \mathrm{H}), 6.75(\mathrm{~s}, 2 \mathrm{H}), 6.85-6.86(\mathrm{~m}, 2 \mathrm{H}), 7.57(\mathrm{~s}$, $4 \mathrm{H}), 7.94$ (br, $\left.4 \mathrm{H}, \mathrm{NH}_{2}\right) ;{ }^{13} \mathrm{C} \mathrm{NMR}\left(100 \mathrm{MHz}\right.$, DMSO- $\left.d_{6}\right): \delta=14.1$, 24.8, 27.1, 27.3, 32.5, 42.8, 44.8, 50.9, 51.1, 79.1, 108.8, 113.3, $122.11,122.7,127.4,127.9,128.1,134.9,135.5,145.3,159.5$, 159.6, 163.5, 168.1, 179.5.

Diethyl-1' 1' $^{\prime \prime \prime}$-(1,4-phenylenebis(methylene))bis(2-amino-7,7dimethyl-2' ${ }^{\prime}$ 5-dioxo-5,6,7,8-tetrahydrospiro[chromene-4,3'indoline]-3-carboxylate) (7t). Cream solid, $\mathrm{mp}>290{ }^{\circ} \mathrm{C} ;{ }^{1} \mathrm{H} \mathrm{NMR}$ $\left(400 \mathrm{MHz}, \mathrm{DMSO}-d_{6}\right): \delta=0.53-0.55(\mathrm{~m}, 6 \mathrm{H}), 0.98(\mathrm{~s}, 6 \mathrm{H}), 1.05(\mathrm{~s}$, $6 \mathrm{H}), 1.26(\mathrm{~s}, 4 \mathrm{H}), 2.11(\mathrm{~s}, 4 \mathrm{H}), 3.40-3.45(\mathrm{~m}, 2 \mathrm{H}), 3.77-3.79(\mathrm{~m}$, $2 \mathrm{H}), 4.71(\mathrm{~d}, J=16 \mathrm{~Hz}, 2 \mathrm{H}) 4.97(\mathrm{~d}, J=16 \mathrm{~Hz}, 2 \mathrm{H}), 6.67$ (d, $J=$ $8 \mathrm{~Hz}, 2 \mathrm{H}), 6.86(\mathrm{~d}, J=8 \mathrm{~Hz}, 2 \mathrm{H}), 6.94-6.95(\mathrm{~m}, 2 \mathrm{H}), 7.06-7.08$ (m, 2H), $7.59(\mathrm{~s}, 4 \mathrm{H}), 7.96\left(\mathrm{br}, 4 \mathrm{H}, \mathrm{NH}_{2}\right) ;{ }^{13} \mathrm{C}$ NMR $(100 \mathrm{MHz}$, DMSO- $\left.d_{6}\right): \delta=14.1,27.2,27.3,32.5,41.9,44.9,51.9,52.0,80.1$, $108.8,113.2$, 122.1, 122.6, 127.4, 127.9, 128.2, 134.9, 136.5, $145.5,159.3,159.5,164.7,168.1,178.7$.

$1^{\prime}, \mathbf{1}^{\prime \prime \prime}$-(1,4-Phenylenebis(methylene))bis(2-amino-7,7-dimethyl2',5-dioxo-5,6,7,8-tetrahydrospiro[chromene-4, $3^{\prime}$-indoline]-3carbonitrile) (7u). Cream solid, $\mathrm{mp}>290{ }^{\circ} \mathrm{C} ;{ }^{1} \mathrm{H}$ NMR $(400 \mathrm{MHz}$, DMSO- $\left.d_{6}\right): \delta=1.04(\mathrm{~s}, 6 \mathrm{H}), 1.07(\mathrm{~s}, 6 \mathrm{H}), 2.12-2.26(\mathrm{~m}, 4 \mathrm{H}), 2.57-$ $2.63(\mathrm{~m}, 4 \mathrm{H}), 4.90(\mathrm{~s}, 4 \mathrm{H}), 6.69-6.71(\mathrm{~m}, 2 \mathrm{H}), 6.98-7.00(\mathrm{~m}, 2 \mathrm{H})$, 7.01-7.15 (m, 4H), 7.34 (br, 4H, $\left.\mathrm{NH}_{2}\right) ;{ }^{13} \mathrm{C}$ NMR $(100 \mathrm{MHz}$, DMSO- $\left.d_{6}\right): \delta=27.5,27.7,28.0,31.2,32.5,43.5,47.2,50.3,56.9$, 110.5 , 111.4, 114.9, 117.8, 126.4, 127.6, 131.5, 135.2, 136.4, $142.4,159.5,165.5,176.8,195.8$.

$1^{\prime}, \mathbf{1}^{\prime \prime \prime}$-(Butane-1,4-diyl)bis(5-amino-8-nitro-2'-oxo-2,3-dihydro-1 $H$-spiro[imidazo[1,2-a]pyridine-7, $3^{\prime}$-indoline]-6-carbonitrile) (8a). Cream powder; $\mathrm{mp}>290{ }^{\circ} \mathrm{C} ;{ }^{1} \mathrm{H} \mathrm{NMR}\left(400 \mathrm{MHz}, \mathrm{CDCl}_{3}\right): \delta=$ $1.75\left(4 \mathrm{H}, \mathrm{m}, \mathrm{CH}_{2} \mathrm{CH}_{2} \mathrm{~N}\right), 3.90\left(4 \mathrm{H}, \mathrm{m}, \mathrm{CH}_{2} \mathrm{~N}\right), 4.15(4 \mathrm{H}, \mathrm{m}$, $\left.\mathrm{CH}_{2} \mathrm{NH}\right), 4.65\left(4 \mathrm{H}, \mathrm{m}, \mathrm{CH}_{2} \mathrm{~N}\right), 6.99$ (2H, d, Ar), 7.44 (4H, m, Ar), 7.87 (2H, d, Ar), 8.4-8.75 (br, $\left.\mathrm{NH}_{2}\right), 9.88$ (2H, s, NH). ${ }^{13} \mathrm{C}$ NMR (100 $\left.\mathrm{MHz}, \mathrm{CDCl}_{3}\right): \delta=29.0,47.3,50.0,51.6,65.4,112.7,117.1,120.5$, 125.2, 127.3, 128.6, 132.5, 138.9, 146.8, 186.8. Anal. calcd for $\mathrm{C}_{34} \mathrm{H}_{30} \mathrm{~N}_{12} \mathrm{O}_{6}$ : C, 58.12; H, 4.03; N, 23.92\%. Found: C, 58.11; H, 4.09; N, 23.93\%.

1,1'-(Hexane-1,6-diyl)bis $\left(6^{\prime}\right.$-amino-9'-nitro-2-oxo-1' $\mathbf{2}^{\prime}, \mathbf{3}^{\prime}, \mathbf{4}^{\prime}$ tetrahydrospiro[indoline-3, $8^{\prime}$-pyrido $[1,2-a]$ pyrimidine $]-7^{\prime}$-carbonitrile) (8b). White powder; $\mathrm{mp}>290{ }^{\circ} \mathrm{C} ;{ }^{1} \mathrm{H}$ NMR $(400 \mathrm{MHz}$, 
$\left.\mathrm{CDCl}_{3}\right): \delta=1.89-2.35\left(16 \mathrm{H}, \mathrm{m}\right.$, aliph), 4.2-4.35 $\left(8 \mathrm{H}, \mathrm{m}, \mathrm{CH}_{2} \mathrm{~N}\right)$, 5.5-6.5 (br, $\mathrm{NH}_{2}$ ), 7.84-7.95 (2H, dd, Ar), 7.95-8.10 (2H, dd, Ar), 8.10-8.25 (2H, dd, Ar), 8.25-8.38 (2H, dd, Ar), $9.94(2 \mathrm{H}, \mathrm{s}, \mathrm{NH})$. ${ }^{13} \mathrm{C} \mathrm{NMR}\left(100 \mathrm{MHz}, \mathrm{CDCl}_{3}\right): \delta=25.7,31.2,32.7,43.2,47.0,48.6$, 50.0, 66.9, 112.7, 120.5, 124.0, 127.3, 128.6, 131.1, 132.5, 138.9, 143.9, 146.8, 186.8. Anal. calcd for $\mathrm{C}_{38} \mathrm{H}_{38} \mathrm{~N}_{12} \mathrm{O}_{6}: \mathrm{C}, 60.15 ; \mathrm{H}$, 5.05 ; N, 22.15\%. Found: C, 60.22; H, 5.06; N, 22.19\%.

$1,1^{\prime \prime}$-(1,4-Phenylenebis(methylene $\left.)\right)$ bis $\left(6^{\prime}\right.$-amino- $3^{\prime}, 3^{\prime}$ dimethyl-9'-nitro-2-oxo- $1^{\prime}, 2^{\prime}, 3^{\prime}, 4^{\prime}$-tetrahydrospiro[indoline-3, $8^{\prime}$ pyrido[1,2-a]pyrimidine]-7'-carbonitrile) (8c). White powder; $\mathrm{mp}>290{ }^{\circ} \mathrm{C} ;{ }^{1} \mathrm{H} \mathrm{NMR}\left(400 \mathrm{MHz}, \mathrm{CDCl}_{3}\right): \delta=1.97\left(12 \mathrm{H}, \mathrm{s}, \mathrm{CH}_{3}\right)$, $3.80\left(8 \mathrm{H}, \mathrm{t}, \mathrm{CH}_{2} \mathrm{~N}\right), 6.82\left(2 \mathrm{H}, \mathrm{d}, \mathrm{CH}_{2} \mathrm{Ar}\right), 7.35$ (8H, m, Ar), 7.43 (4H, d, Ar), 9.70-10.10 (br, $\left.\mathrm{NH}_{2}\right), 10.16(2 \mathrm{H}, \mathrm{s}, \mathrm{NH}) .{ }^{13} \mathrm{C} \mathrm{NMR}$ $\left(100 \mathrm{MHz} \mathrm{CDCl}_{3}\right): \delta=23.9,36.5,42.3,53.6,55.2,61.1,112.3$, $115.2,120.3,123.8,127.4,129.0,129.6,133.5,136.3,137.3$, 143.0, 150.8, 154.1, 177.1. Anal. calcd for $\mathrm{C}_{44} \mathrm{H}_{42} \mathrm{~N}_{12} \mathrm{O}_{6}$ : C, 63.30; H, 5.07; N, 20.13\%. Found: C, 63.30; H, 5.09; N, 22.19\%.

1,1' $1^{\prime \prime}$-(Butane-1,4-diyl)bis (6'-amino-5,9'-dinitro-2-oxo- $\mathbf{1}^{\prime}, 2^{\prime}$, $3^{\prime}, 4^{\prime}$-tetrahydrospiro[indoline-3,8 $8^{\prime}$-pyrido $[1,2-a]$ pyrimidine $]-7^{\prime}$ carbonitrile) (8d). Pale yellow powder; $\mathrm{mp}>290{ }^{\circ} \mathrm{C} ;{ }^{1} \mathrm{H}$ NMR $\left(400 \mathrm{MHz}, \mathrm{CDCl}_{3}\right): \delta=1.41-1.57(4 \mathrm{H}, \mathrm{m}$, aliph), $1.80-2.02(4 \mathrm{H}$, $\mathrm{m}$, aliph), 2.92-3.07 (4H, m, $\left.\mathrm{CH}_{2} \mathrm{~N}\right), 3.17-3.26\left(4 \mathrm{H}, \mathrm{m}, \mathrm{CH}_{2} \mathrm{~N}\right)$, 3.81-3.94 (4H, m, $\left.\mathrm{CH}_{2} \mathrm{~N}\right), 7.54-7.6(4 \mathrm{H}, \mathrm{m}, \mathrm{Ar}), 7.94-8.01(2 \mathrm{H}, \mathrm{d}$, $\mathrm{Ar}), 9.8(2 \mathrm{H}, \mathrm{d}, \mathrm{NH}) .{ }^{13} \mathrm{C} \mathrm{NMR}\left(100 \mathrm{MHz}, \mathrm{CDCl}_{3}\right): \delta=23.5,25.5$, 42.2, 49.5, 115.7, 116.1, 116.9, 129.2, 133.5, 137.1, 140.1, 143.8, 150.7, 154.5, 175.6. Anal. calcd for $\mathrm{C}_{36} \mathrm{H}_{32} \mathrm{~N}_{14} \mathrm{O}_{10}: \mathrm{C}, 52.68 ; \mathrm{H}$, 3.93; N, 23.89\%. Found: C, 52.72; H, 3.93; N, 23.94\%.

1,1'-(Hexane-1,6-diyl)bis ( $2^{\prime}$-amino-1' -benzyl-6' -(benzylamino)-5, $5^{\prime}$-dinitro-2-oxo-1' $H$-spiro[indoline-3, $4^{\prime}$-pyridine $]-3^{\prime}$ carbonitrile) (8e). Yellow powder; $\mathrm{mp}>290{ }^{\circ} \mathrm{C} ;{ }^{1} \mathrm{H}$ NMR $(400$ $\left.\mathrm{MHz} \mathrm{CDCl}_{3}\right): \delta=0.70-0.82(4 \mathrm{H}, \mathrm{m}$, aliph), 1.85-1.94 $(4 \mathrm{H}, \mathrm{m}$, aliph), 4.04-4.12 (4H, m, $\left.\mathrm{CH}_{2} \mathrm{~N}\right), 6.26-6.33\left(2 \mathrm{H}, \mathrm{t}, \mathrm{CH}_{2} \mathrm{Ar}\right), 7.45-$ 7.51 (20H, m, Ar), 7.53-7.59 (2H, m, Ar), 8.5-9.5 (br, $\mathrm{NH}_{2}$ ), 9.98 $(2 \mathrm{H}, \mathrm{d}, \mathrm{NH}) .{ }^{13} \mathrm{C}$ NMR $\left(100 \mathrm{MHz}, \mathrm{CDCl}_{3}\right): \delta=26.7,27.6,42.4$, 48.6, 49.4, 53.2, 115.2, 116.0, 117.0, 127.1, 127.6, 128.4, 128.9, 129.2 , 133.7, 136.8, 137.2, 139.0, 140.2, 143.2, 150.6, 155.4, 174.8. Anal. calcd for $\mathrm{C}_{60} \mathrm{H}_{52} \mathrm{~N}_{14} \mathrm{O}_{10}: \mathrm{C}, 63.82 ; \mathrm{H}, 4.64 ; \mathrm{N}$, $17.37 \%$. Found: C, 63.86; H, 4.61; N, 17.34\%.

$1^{\prime}, 1^{\prime \prime \prime}$-(1,4-Phenylenebis(methylene))bis(5-amino- ${ }^{\prime}, 8$ dinitro-2'-oxo-2,3-dihydro-1H-spiro[imidazo[1,2-a]pyridine-7, $3^{\prime}$ indoline]-6-carbonitrile) (8f). Cream powder; $\mathrm{mp}>290{ }^{\circ} \mathrm{C} ;{ }^{1} \mathrm{H}$ NMR (400 MHz, $\left.\mathrm{CDCl}_{3}\right): \delta=3.89-4.00\left(4 \mathrm{H}, \mathrm{dt}, \mathrm{CH}_{2} \mathrm{~N}\right), 4.08-4.21$ $\left(4 \mathrm{H}, \mathrm{dt}, \mathrm{CH}_{2} \mathrm{NH}\right), 6.34\left(4 \mathrm{H}, \mathrm{dd}, \mathrm{CH}_{2} \mathrm{Ar}\right), 6.69(2 \mathrm{H}, \mathrm{d}, \mathrm{Ar}), 7.16(2 \mathrm{H}$, d, Ar), 7.29 (4H, m, Ar), 7.40 (2H, t, Ar), 8.91-9.52 (br, $\left.\mathrm{NH}_{2}\right), 9.98$ $(2 \mathrm{H}, \mathrm{d}, \mathrm{NH}) .{ }^{13} \mathrm{C} \mathrm{NMR}\left(100 \mathrm{MHz}, \mathrm{CDCl}_{3}\right): \delta=42.4,42.9,48.7$, 53.1, 115.8, 116.3, 117.0, 127.7, 128.2, 133.8, 136.4, 137.1, 140.7, 143.2, 150.3, 154.4, 175.9. Anal. calcd for $\mathrm{C}_{38} \mathrm{H}_{28} \mathrm{~N}_{14} \mathrm{O}_{10}: \mathrm{C}$, 54.29; H, 3.36; N, 23.32\%. Found: C, 54.35; H, 3.31; N, 23.38\%.

1,1' $1^{\prime \prime}$-(Hexane-1,6-diyl)bis ( $6^{\prime}$-amino- $3^{\prime}, 3^{\prime}, 5$-trimethyl-9' -nitro2-oxo-1' $, 2^{\prime}, 3^{\prime}, 4^{\prime}$-tetrahydrospiro[indoline-3, $8^{\prime}$-pyrido $[1,2-a]$ pyrimidine]-7'-carbonitrile) (8g). White powder; $\mathrm{mp}>290{ }^{\circ} \mathrm{C} ;{ }^{1} \mathrm{H}$ NMR (400 MHz, $\left.\mathrm{CDCl}_{3}\right): \delta=0.81\left(4 \mathrm{H}, \mathrm{m}, \mathrm{CH}_{2}\right), 0.91(6 \mathrm{H}, \mathrm{s}, \mathrm{Me})$, $1.04(6 \mathrm{H}, \mathrm{s}, \mathrm{Me}), 1.19$ (4H, m, aliph), $2.93(6 \mathrm{H}, \mathrm{s}, \mathrm{MeAr}), 4.04(4 \mathrm{H}$, d, $\left.\mathrm{NCH}_{2}\right), 4.26\left(4 \mathrm{H}, \mathrm{d}, \mathrm{NHCH}_{2}\right), 4.55\left(4 \mathrm{H}, \mathrm{m}, \mathrm{CH}_{2} \mathrm{~N}\right), 7.08(2 \mathrm{H}, \mathrm{d}$, $\mathrm{Ar}), 7.20$ (2H, t, Ar), 7.31 (2H, t, Ar), 10.22 (2H, d, NH). ${ }^{13} \mathrm{C} \mathrm{NMR}$ $\left(100 \mathrm{MHz}, \mathrm{CDCl}_{3}\right): \delta=20.1,23.1,26.4,27.0,36.9,42.1$, 49.0, 55.8, 61.2, 115.0, 115.6, 126.3, 127.1, 128.1, 129.2, 133.8, 134.7,
137.6, 143.8, 150.1, 154.5, 174.8. Anal. calcd for $\mathrm{C}_{44} \mathrm{H}_{50} \mathrm{~N}_{12} \mathrm{O}_{6}: \mathrm{C}$, 62.69; H, 5.98; N, 19.94\%. Found: C, 62.72; H, 6.04; N, 20.00\%.

$1^{\prime}, \mathbf{1}^{\prime \prime \prime}$-(1,2-Phenylenebis(methylene))bis(5-amino-5'-methyl8-nitro-2'-oxo-2,3-dihydro-1 $H$-spiro[imidazo[1,2- $a$ ]pyridine-7, $3^{\prime}$ indoline]-6-carbonitrile) (8h). Cream powder; $\mathrm{mp}>290{ }^{\circ} \mathrm{C} ;{ }^{1} \mathrm{H}$ NMR (400 MHz, $\left.\mathrm{CDCl}_{3}\right): \delta=3.03(6 \mathrm{H}, \mathrm{s}, \mathrm{Me}), 3.77(8 \mathrm{H}, \mathrm{m}$, $\left.\mathrm{CH}_{2} \mathrm{~N}\right), 6.78$ (4H, d, $\left.\mathrm{CH}_{2} \mathrm{Ar}\right), 7.10$ (2H, t, Ar), 7.17 (2H, d, Ar), 7.29 (2H, t, Ar), 7.40 (2H, t, Ar), 8.70-9.30 (br, $\left.\mathrm{NH}_{2}\right), 9.78(2 \mathrm{H}, \mathrm{d}, \mathrm{NH})$. ${ }^{13} \mathrm{C}$ NMR (100 MHz, $\left.\mathrm{CDCl}_{3}\right): \delta=21.6,42.1,42.7,50.2,53.0$, $115.3,117.2$, 126.8, 127.1, 127.6, 128.7, 129.3, 131.6, 133.0, 134.1, 137.4, 143.3, 150.3, 155.2, 174.4. Anal. calcd for $\mathrm{C}_{40} \mathrm{H}_{34} \mathrm{~N}_{12} \mathrm{O}_{6}$ : C, 61.69; H, 4.40; N, 21.58\%. Found: C, 61.61; H, 4.35 ; N, 21.58\%.

1,1"-(1,4-Phenylenebis(methylene))bis(6'-amino-5-methyl-9'-

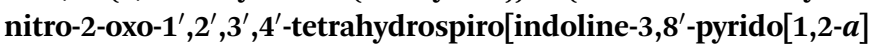
pyrimidine]-7'-carbonitrile) (8i). Yellow powder; $\mathrm{mp}>290{ }^{\circ} \mathrm{C}$; $\left.{ }^{1} \mathrm{H} \mathrm{NMR} \mathrm{(400} \mathrm{MHz,} \mathrm{CDCl}_{3}\right): \delta=1.65(4 \mathrm{H}, \mathrm{m}$, aliph), $3.59(4 \mathrm{H}, \mathrm{t}$, $\left.\mathrm{CH}_{2} \mathrm{~N}\right), 3.73\left(4 \mathrm{H}, \mathrm{t}, \mathrm{CH}_{2} \mathrm{NH}\right), 3.85\left(6 \mathrm{H}, \mathrm{s}, \mathrm{CH}_{3}\right), 6.47(2 \mathrm{H}, \mathrm{d}$, $\mathrm{CH}_{2} \mathrm{Ar}$ ), 7.05 (2H, dt, Ar), 7.15 (2H, m, Ar), 7.35 (2H, dt, Ar), 7.45 (2H, t, Ar), 7.54 (2H, dt, Ar), 10.03 (2H, s, NH). ${ }^{13} \mathrm{C}$ NMR (100 $\left.\mathrm{MHz} \mathrm{CDCl}_{3}\right): \delta=18.1,20.1,23.8,42.6,48.3,49.0,53.2,115.8$, 117.1 , 127.7, 127.9, 128.8, 129.0, 133.7, 134.4, 136.1, 137.2, 143.7, 150.3, 153.3, 154.0, 175.6. Anal. calcd for $\mathrm{C}_{42} \mathrm{H}_{38} \mathrm{~N}_{12} \mathrm{O}_{6}: \mathrm{C}$, 62.65 ; H, 4.75; N, 20.83\%. Found: C, 62.61; H, 4.72; N, 20.79\%.

1,1' ${ }^{\prime \prime}$-(Butane-1,4-diyl)bis( $6^{\prime}$-amino-5-fluoro- $\mathbf{7}^{\prime}$-isocyano-9' nitro-1 ${ }^{\prime}, 2^{\prime}, 3^{\prime}, 4^{\prime}$-tetrahydrospiro[indoline-3, $8^{\prime}$-pyrido $[1,2-a]$ pyrimidin]-2-one) (8j). Cream powder; $\mathrm{mp}>290{ }^{\circ} \mathrm{C} ;{ }^{1} \mathrm{H}$ NMR $\left(400 \mathrm{MHz}, \mathrm{CDCl}_{3}\right): \delta=0.87$ (4H, m, aliph), 1.01 (4H, m, aliph), $4.23\left(8 \mathrm{H}, \mathrm{m}, \mathrm{CH}_{2} \mathrm{~N}\right), 4.50\left(4 \mathrm{H}, \mathrm{t}, \mathrm{NCH}_{2}\right), 7.25(2 \mathrm{H}, \mathrm{d}, \mathrm{Ar}), 7.58$ $(2 \mathrm{H}, \mathrm{d}, \mathrm{Ar}), 7.70$ (2H, t, Ar), $10.03(2 \mathrm{H}, \mathrm{s}, \mathrm{NH}) .{ }^{13} \mathrm{C}$ NMR (100 $\left.\mathrm{MHz}, \mathrm{CDCl}_{3}\right): \delta=23.7,25.5,38.0,42.3,49.8,114.2,114.7,117.2$, 133.5, 136.8, 137.2, 143.9, 150.6, 161.1, 161.4, 176.3. Anal. calcd for $\mathrm{C}_{36} \mathrm{H}_{32} \mathrm{~F}_{2} \mathrm{~N}_{12} \mathrm{O}_{6}$ : C, 56.39; $\mathrm{H}, 4.21 ; \mathrm{N}, 21.92 \%$. Found: C, $56.49 ; \mathrm{H}, 4.28$; N, 21.86\%.

1,1'-(1,4-Phenylenebis(methylene))bis(2'-amino-1' -benzyl-6' (benzylamino)-5-fluoro-5' ${ }^{\prime}$ nitro-2-oxo-1' $H$-spiro[indoline-3, $\mathbf{4}^{\prime}$ pyridine]-3'-carbonitrile) (8k). White powder; $\mathrm{mp}>290{ }^{\circ} \mathrm{C} ;{ }^{1} \mathrm{H}$ NMR (400 MHz, $\left.\mathrm{CDCl}_{3}\right): \delta=7.76\left(8 \mathrm{H}, \mathrm{dd}, \mathrm{CH}_{2} \mathrm{Ar}\right), 6.28(4 \mathrm{H}, \mathrm{t}$, $\mathrm{CH}_{2} \mathrm{Ar}$ ), 7.21 (2H, d, Ar), 7.34 (2H, d, Ar), 7.43-7.60 (20H, m, Ar), 7.57 (2H, t, Ar), 7.92 (4H, m, Ar), 8.60-9.10 (br, $\left.\mathrm{NH}_{2}\right), 10.00(2 \mathrm{H}$, d, NH). ${ }^{13} \mathrm{C}$ NMR (100 MHz, $\left.\mathrm{CDCl}_{3}\right): \delta=19.0,42.0,49.3,49.5$, 53.2, 114.2, 114.6, 115.6, 117.6, 127.4, 127.7, 128.1, 128.5, 129.2, 133.8, 136.3, 136.9, 139.5, 143.2, 150.8, 153.6, 154.0, 161.2, 173.9. Anal. calcd for $\mathrm{C}_{62} \mathrm{H}_{48} \mathrm{~F}_{2} \mathrm{~N}_{12} \mathrm{O}_{6}: \mathrm{C}, 68.00 ; \mathrm{H}, 4.42 ; \mathrm{N}$, $15.35 \%$. Found: C, 68.03; H, 4.36; N, 15.41\%.

$1^{\prime}, \mathbf{1}^{\prime \prime \prime}$-(Butane-1,4-diyl)bis(5-amino-5' -bromo-6-isocyano-8nitro-2,3-dihydro-1H-spiro[imidazo[1,2-a]pyridine-7, $\mathbf{3}^{\prime}$-indolin]2'-one) (81). White powder; $\mathrm{mp}>290{ }^{\circ} \mathrm{C} ;{ }^{1} \mathrm{H}$ NMR $(400 \mathrm{MHz}$, $\left.\mathrm{CDCl}_{3}\right): \delta=1.15\left(4 \mathrm{H}, \mathrm{m}\right.$, aliph), $3.94\left(4 \mathrm{H}, \mathrm{m}, \mathrm{CH}_{2} \mathrm{~N}\right), 4.13(4 \mathrm{H}, \mathrm{m}$, $\left.\mathrm{CH}_{2} \mathrm{NH}\right), 4.40\left(4 \mathrm{H}, \mathrm{m}, \mathrm{CH}_{2} \mathrm{~N}\right), 7.2-7.6\left(\mathrm{br}, \mathrm{NH}_{2}\right), 7.70-7.85(4 \mathrm{H}$, m, Ar), 7.93-7.99 (2H, m, Ar), 9.77 (1 H, s, NH). ${ }^{13} \mathrm{C}$ NMR (100 $\left.\mathrm{MHz}, \mathrm{CDCl}_{3}\right): \delta=25.5,42.2,42.7,48.8,49.8,117.2,117.7,126.2$, 133.0, 134.6, 136.4, 137.1, 143.0, 151.6, 160.2, 175.5. Anal. calcd for $\mathrm{C}_{34} \mathrm{H}_{28} \mathrm{Br}_{2} \mathrm{~N}_{12} \mathrm{O}_{6}$ : C, 47.46; H, 3.28; N, 19.53\%. Found: C, $47.40 ; \mathrm{H}, 3.25$; N, 19.46\%.

1,1"-(1,2-Phenylenebis(methylene))bis(6'-amino-5-bromo$3^{\prime}, 3^{\prime}$-dimethyl-9' -nitro-2-oxo-1 ${ }^{\prime}, 2^{\prime}, 3^{\prime}, 4^{\prime}$-tetrahydrospiro[indoline- 
3,8'-pyrido[1,2-a]pyrimidine]-7'-carbonitrile) (8m). Yellow powder; mp > $290{ }^{\circ} \mathrm{C} ;{ }^{1} \mathrm{H}$ NMR $\left(400 \mathrm{MHz}, \mathrm{CDCl}_{3}\right): \delta=1.80$ $\left(6 \mathrm{H}, \mathrm{s}, \mathrm{CH}_{3}\right), 1.85\left(6 \mathrm{H}, \mathrm{s}, \mathrm{CH}_{3}\right), 3.45\left(4 \mathrm{H}, \mathrm{m}, \mathrm{CH}_{2} \mathrm{~N}\right), 3.83(4 \mathrm{H}, \mathrm{m}$, $\left.\mathrm{CH}_{2} \mathrm{NH}\right), 4.70-5.04$ (4H, dd, $\left.\mathrm{CH}_{2} \mathrm{Ar}\right), 7.29(2 \mathrm{H}, \mathrm{t}, \mathrm{Ar}), 7.47(2 \mathrm{H}, \mathrm{d}$, $\mathrm{Ar}), 7.76$ (2H, t, Ar), 8.06 (2H, d, Ar), 8.22 (2H, d, Ar), 9.90-10.20 (br, $\left.\mathrm{NH}_{2}\right), 10.26(2 \mathrm{H}, \mathrm{s}, \mathrm{NH}) .{ }^{13} \mathrm{C} \mathrm{NMR}\left(100 \mathrm{MHz}, \mathrm{CDCl}_{3}\right): \delta=$ 23.8, 36.6, 42.8, 53.2, 55.1, 61.3, 115.1, 117.1, 117.5, 126.2, 127.6, 129.5, 131.1, 133.4, 134.5, 137.5, 143.1, 151.2, 151.5, 175.5. Anal. calcd for $\mathrm{C}_{44} \mathrm{H}_{40} \mathrm{Br}_{2} \mathrm{~N}_{12} \mathrm{O}_{6}$ : C, 53.24; H, 4.06; N, 16.93\%. Found: C, 53.16; H, 4.11; N, 16.91\%.

1,1' ${ }^{\prime \prime}$-(1,4-Phenylenebis(methylene))bis (2' -amino-1' -benzyl-6' (benzylamino)-5-bromo-5' -nitro-2-oxo-1' $H$-spiro[indoline-3, $4^{\prime}$ pyridine]-3'-carbonitrile) (8n). Cream powder; $\mathrm{mp}>290{ }^{\circ} \mathrm{C} ;{ }^{1} \mathrm{H}$ NMR (400 MHz, $\left.\mathrm{CDCl}_{3}\right): \delta=6.00\left(8 \mathrm{H}, \mathrm{dd}, \mathrm{CH}_{2} \mathrm{Ar}\right), 6.28(4 \mathrm{H}, \mathrm{dt}$, $\left.\mathrm{CH}_{2} \mathrm{Ar}\right), 7.15$ (12H, m, Ar), 7.42 (4H, m, Ar), 7.63 (2H, t, Ar), 7.88 $(2 \mathrm{H}, \mathrm{t}, \mathrm{Ar}), 8.02$ (2H, d, Ar) $8.21(4 \mathrm{H}, \mathrm{m}, \mathrm{Ar}), 8.31(2 \mathrm{H}, \mathrm{d}, \mathrm{Ar})$, 10.11 (2H, d, NH). ${ }^{13} \mathrm{C}$ NMR (100 MHz, $\left.\mathrm{CDCl}_{3}\right): \delta=20.6,43.0$, 48.4, 48.7, 53.4, 115.4, 117.1, 117.6, 126.3, 127.2, 127.7, 128.5, $128.9,129.5$, 133.1, 134.4, 136.4, 139.5, 143.7, 150.3, 153.6, 153.9, 174.4. Anal. calcd for $\mathrm{C}_{62} \mathrm{H}_{48} \mathrm{Br}_{2} \mathrm{~N}_{12} \mathrm{O}_{6}$ : C, 61.19; H, 3.98; N, 13.81\%. Found: C, 61.16; H, 4.03; N, 13.86\%.

\section{Conclusions}

In summary, a highly efficient and green method for one-pot multi-component synthesis of bis-spirooxindoles has been reported. The described procedure takes advantage of PEG-400 as an inexpensive, biodegradable, commercially available and reusable solvent. Non-toxic and environmentally friendly reagent, $\mathrm{K}_{2} \mathrm{CO}_{3}$, was used to promote the reaction toward completion. The generality of the method has been investigated using ketene aminals as well as enolizable ketones to form a library of complex structure of bis-spiro compounds.

\section{Conflicts of interest}

There are no conflicts to declare.

\section{Acknowledgements}

The authors thank Persian Gulf University Research Councils for the financial support of this work.

\section{References}

1 R. L. Swann and A. Eschenroeder, Fate of chemicals in the environment, ACS Symposium sries, Washington DC, 1983, vol. 225.

2 A. Sarkar, S. Santra, S. K. Kundu, A. Hajra, G. V. Zyryanov, O. N. Chupakhin, V. N. Charushin and A. Majee, Green Chem., 2016, 18, 4475-4525.

3 (a) D. J. C. Constable, A. D. Curzons and V. L. Cunningham, Green Chem., 2002, 4, 521-527; (b) A. D. Curzons, D. J. C. Constable, D. N. Mortimer and V. L. Cunningham, Green Chem., 2001, 3, 1-6.

4 H. R. Safaei, M. Shekouhy, S. Rahmanpur and A. Shirinfeshan, Green Chem., 2012, 14, 1696.
5 J. Song and B. Han, Natl. Sci. Rev., 2015, 1-2.

6 C. Capello, U. Fisher and K. Kungerbühler, Green Chem., 2007, 9, 927-934.

7 A. Chanda and V. V. Fokin, Chem. Rev., 2009, 109, 725-748.

8 (a) J. Chen, S. K. Spear, J. G. Huddleston and R. D. Rogers, Green Chem., 2005, 7, 64-82; (b) J. Bi, Z. Zhang, Q. Liu and G. Zhang, Green Chem., 2012, 14, 1159; (c) J. M. Khurana, A. Chaudhary, A. Lumb and B. Nand, Green Chem., 2012, 14, 2321; (d) Y. Zou, Q. Huang, T. K. Huang, Q. C. Ni, E. S. Zhang, T. L. Xu, M. Yuand and J. Lie, Org. Biomol. Chem., 2013, 11, 6967; (e) G. D. Yadav and B. G. Motirale, Org. Process Res. Dev., 2009, 13, 341; $(f)$ M. Vafaeezadeh and M. Mahmoodi Hashemi, J. Mol. Liq., 2015, 207, 73-79.

9 C. C. A. Cariou, G. J. Clarkson and M. Shipman, J. Org. Chem., 2008, 73, 9762-9764.

10 L. Zhou, D. S. Bohle, H. F. Jiang and C. J. Li, Synlett, 2009, 937-940.

11 G. Ren, J. Zhang, Z. Duan, M. Cui and Y. Wu, Aust. J. Chem., 2009, 62, 75-81.

12 S. L. Schreiber, Science, 2000, 287, 1964-1969.

13 R. C. Cioc, E. Ruijter and R. V. A. Orru, Green Chem., 2014, 16, 2958-2975.

14 M. Zolfigol, A. Khazaei, A. Zare, M. Mokhlesi, T. HekmatZadeh, A. Hasaninejad, F. Derakhshan-Panah, A. MoosaviZare, H. Keypour, A. Deghani-Firouzabadi and M. Merajoddin, J. Chem. Sci., 2012, 124, 501-508.

15 A. Zare, R. Khanivar, M. Hatami, M. Mokhlesi, M. Zolfigol, A. Moosavi-Zare, A. Hasaninejad, A. Khazaei and V. Khakyzadeh, J. Mex. Chem. Soc., 2012, 4, 56.

16 A. Hasaninejad, S. Firoozi and F. Mandegani, Tetrahedron Lett., 2013, 54, 2791-2794.

17 (a) P. Helene, Adv. Synth. Catal., 2012, 354, 237-294; (b) J. G. Hernandez and E. Juaristi, Chem. Commun., 2012, 48, 5396-5409; (c) D. Bonne, T. Constantieux, Y. Coquerel and J. Rodriguez, Org. Biomol. Chem., 2012, 10, 3969-3973.

18 Y. Gu, Green Chem., 2012, 14, 2091-2128.

19 G. R. Khabibullin, E. S. Fedotov, V. R. Akhmetov, E. S. Mesheryakov, L. M. Khalilov and A. G. bragimov, Mol. Diversity, 2016, 2, 557-565.

20 S. K. Panja and S. Saha, RSC Adv., 2013, 3, 14495-14500.

21 A. Maryamabadi, A. Hasaninejad, N. Nowrouzi, G. H. Mohebbi and B. Asghari, Bioorg. Med. Chem., 2016, 6, 1408-1417.

22 P. Licence, J. Ke, M. Sokolova, S. K. Rossb and M. Poliakoff, Green Chem., 2003, 5, 99-104.

23 A. Dandia, A. K. Jain and A. K. Laxkar, Tetrahedron Lett., 2013, 30, 3929-3932.

24 R. A. Sheldon, Green Chem., 2005, 7, 267-287.

25 A. Hasaninejad, A. Zare, M. Shekouhya and J. Ameri-Rada, Green Chem., 2011, 13, 958-964.

26 R. Sakhuja, S. S. Panda, L. Khanna, S. Khurana and S. C. Jain, Bioorg. Med. Chem., 2011, 21, 5465-5469.

27 K. Parthasarathy, C. Praveen, C. Balachandran, p. S. Kumar, S. Ignacimuthu and P. T. Perumal, Bioorg. Med. Chem., 2013, 23, 2708-2713.

28 A. Maryamabadi, A. Hasaninejad, N. Nowrouzi and G. H. Mohebbi, Bioorg. Med. Chem., 2017, 25, 2057-2064. 
29 P. Khanna, A. Saxena, L. Khanna, S. Bhagat and S. C. Jain, ARKIVOC, 2009, 5, 119-125.

30 M. Jain, R. Sakhuja, P. j. Khanna, S. Bhagat and S. C. Jaina, ARKIVOC, 2008, 15, 54-64.

31 C. M. Clay, H. M. Abdallah, C. Jordan, K. Knisley and D. M. Ketcha, ARKIVOC, 2012, 6, 317-325.

32 M. Kidwai, A. Jain, V. Nemaysh, R. Kumar and P. Luthra, Med. Chem. Res., 2013, 22, 2717-2723.

33 (a) J. Chen, S. K. Spear, J. G. Huddleston and R. D. Rogers, Green Chem., 2005, 7, 64-82; (b) D. Q. Xu, S. P. Luo, Y. F. Wang, A. B. Xia, H. D. Yue, L. P. Wang and Z. Y. Xu, Chem. Commun., 2007, 42, 4393-4395; (c) Y. N. Li, J. L. Wang and L. N. He, Tetrahedron Lett., 2011, 52, 34853488.

34 R. Ballini, G. Bosica, M. L. Conforti, R. Magg, A. Mazzacani, P. Righi and G. Sartori, Tetrahedron, 2001, 7, 1395-1398.

35 K. Hiramoto, A. Nasuhara, K. Michikoshi, T. Kato and K. Kikugawa, Mutat. Res., Genet. Toxicol. Environ. Mutagen., 1997, 395, 47-56.
36 G. Khanna, K. Aggarwal and J. M. Khurana, Synth. Commun., 2016, 23, 1880-1886.

37 J. Ma and S. M. Hecht, Chem. Commun., 2004, 1190-1191.

38 C. B. Cui, H. Kakeya and H. Osada, Tetrahedron, 1996, 52, 12651-12666.

39 T. Usui, M. Kondoh, C. B. Cui, T. Mayumi and H. Osada, Biochem. J., 1998, 333, 543-548.

40 J. F. M. Silva and A. C. Pinto, J. Braz. Chem. Soc., 2001, 12, 273-324.

41 L. R. Wen, C. L. Mingli and L. J. Wang, J. Org. Chem., 2010, 75, 7605-7614.

42 D. P. Sangi, J. L. Monteiro, K. L. Vanzolini, Q. B. Cass, M. W. Paixao and A. G. Corrêa, J. Braz. Chem. Soc., 2014, 25, 887-889.

43 K. M. Wang and S. J. Yan, Eur. J. Org. Chem., 2014, 6, 11291145.

44 X. Wang, Z. Quan and Z. Zhang, Tetrahedron, 2007, 63, 82278233. 